\title{
Principales características de la agricultura en los estados fronterizos: evolución del patrón de cultivos
}

\author{
Rosario Pérez Espejo
}

\section{Resumen}

Este trabajo tiene como objetivo señalar algunas de las características más relevantes de la agricultura en los estados de la frontera norte, poniendo especial énfasis en los cambios ocurridos en el patrón de cultivos en un período que va de 1960 a 1983. El trabajo fue dividido en tres apartados: en el primero se señalan las características generales del sector atendiendo a tres elementos con una tendencia común en la agricultura mexicana. En el segundo apartado se analiza la evolución al interior del sector a través del uso del suelo realizada por la agricultura y la ganadería; y en el último apartado se presenta la evolución de la agricultura con base en su participación dentro de la agricultura nacional y los cambios presentados en el patrón de cultivos.

Palabras clave: patrón de cultivo, agricultura, ganadería, uso del suelo, estados fronterizos de México.

\begin{abstract}
The objective of this work is to outline some of the most outstanding characteristics of agriculture in the states from the Northern border, emphasizing on changes suffered by cultivation patterns during the period of time between 1960 and 1983. This project was divided in three parts: the first one points out the general characteristics of the sector based on three elements that have a common trend on Mexican agriculture. The second part analyzes the evolution to the interior of the sector performed by agriculture and cattle through the use of soil. And finally, it presents the evolution of agriculture based on its participation within domestic agriculture as well as the changes presented by cultivation patterns.
\end{abstract}

Keywords: cultivation patterns, agriculture, cattle, use of soil, Mexican border states. 


\title{
PRINCIPALES CARACTERISTICAS DE LA AGRICULTURA EN LOS ESTADOS FRONTERIZOS: EVOLUCION DEL PATRON DE CULTIVOS
}

\author{
Por \\ Rosario Perez Espejo
}

\section{PRESENTACION.}

Nuestro trabajo tiene como objetivo señalar en forma somera algunas de las características más relevantes de la agricultura en los estados de la frontcra norte, poniendo especial énfasis en los cambios ocurridos en el patrón de cultivos en un período que va de 1960 a 1983 ?

Los estudios sobre regionalización consideran como unidad fronteriza un área que abarca zonas de seis entidades - Baja California, Coahuila, Chihuahua, Nuevo León, Sonora y Tamaulipas-, que de acuerdo con la división geoeconómica del país se encuentran dentro de tres grandes regiones: noroeste, norte y noreste. Forman parte de la zona fronteriza 60 municipios que están totalmente ligados a la unidad político-administrativa que es la línea fronteriza. ${ }^{2}$

Las regiones que integran la frontera norte presentan elementos de diferenciación integral y características contrastantes tanto en los aspectos físicos como en los económicos. De allí que exista una gran heterogeneidad entre estas regiones, misma que se acentúa al tomar como unidad de análisis a la entidad federativa -como es el caso de este trabajo-y no al municipio.

No obstante sus especificidades, regiones y estados fronterizos comparten características comunes entre sí y con el resto del país, y los problemas políticos, sociales y económicos que distinguen a la agricultura mexicana se presentan en mayor o menor grado en dichos espacios.

El desempleo rural, la incquitativa distribución del ingreso entre sectores, los problemas en torno a la tenencia de la tierra, la inadecuada estructura del comercio de los productos agropecuarios, la lucha de clases en el campo son, entre otros, componentes de una problemática rural presente en to-

\footnotetext{
${ }^{1}$ Se seleccionaron 33 cultivos y seis observaciones: $1960,1965,1971,1977,1979,1983$. La cobertura fue de $97.6 \%, 96.0 \%, 79.0 \%, 92.0 \%, 92.9 \%$ y $94.8 \%$ en cada uno de las observaciones en re lación con la superficie cosechada total. La fuente de información fue la Dirección General de Econo. mía Agrícola de la SARH.

2 Analisis espacial de la frontera norte de México, Javier Delgadillo, tesis, Facultad de Filosofía y Le. tras. UNAM, 1982. Algunos autores consideran dentro de esta zona sólo a los 34 municipios lim ftrofes. Ver: Nuestra dependencia fronteriza: Joaquín Xirau y Miguel Díaz. F.C.E., México, 1976.
} 
das las regiones del país y los estados de la frontera norte no son una excepción a esta regla.

En términos generales, los problemas aludidos son el resultado de un proceso histórico en el cual el proceso de acumulación de capital ha subordinado las actividades agrícolas al resto de la economía. A este proceso de carácter interno se agrega la influencia cada vez más patente del desarrollo de la acumulación a nivel internacional que conforma una división internacional de trabajo en la cual se inserta México como país subdesarrollado y dependiente.

Hemos dividido el trabajo en tres apartados: en el primero se señalan las características generales del sector atendiendo a tres elementos cuya tendencia es común en la agricultura mexicana: La evolución del producto interno bruto agrícola en relación al producto total; la evolución de la población económicamente activa en el sector y su participación en el ingreso generado. En el segundo apartado se analiza la evolución al interior del sector a través del uso del suelo, realizado por parte de la agricultura y la ganadería; y en el último apartado se presenta la evolución de la agricultura con base en su participación dentro de la agricultura nacional y los cambios que se han presentado en el patrón de cultivos.

\section{LAS ACTIVIDADES AGROPECUARIAS EN LA FRONTERA NORTE.}

\section{Evolución del PIB.}

La participación del valor de la producción agropecuaria de los estados de la frontera en el PIB agropecuario nacional ha sido sorprendentemente estable a lo largo de 30 años, representando el $22 \%$ tanto en 1950 como en 1980 .

La década de los cincuentas representó un período de gran dinamismo de las actividades agropecuarias en la mayor parte del país. En los estados de Sonora y Chihuahua, el auge del sector reflejado en un incremento de la participación del PIB agropecuario dentro del PIB total estatal de 1950 a 1960 , estuvo sustentado en la producción de algodón en el primer caso y en la producción ganadera en el segundo. En los demás estados de la frontera, la reducción relativa del sector agropecuario dentro de la economía es la regla común. En 1980 más del 50\% de la producción se genera en los sectores de servicios y distribución; sólo en Nuevo León, un importante monto de la producción es obtenida en la industria manufacturera (36.8\%). El sector agropecuario en ese año es todavía importante en Sonora ( $20 \%$ del PIB); en menor medida en Chihuahua (14\%) y en Tamaulipas (10\%) (ver cuadros I y II). 
CUADRO I. Estados Fronterizos: Porcentaje del PIB agropecuario en relación al total. 1950, 1960, 1980.

\begin{tabular}{lrrr}
\hline & $\mathbf{1 9 5 0}$ & $\mathbf{1 9 6 0}$ & $\mathbf{1 9 8 0}$ \\
\hline Baja California & 21.5 & 17.4 & 9.5 \\
Coahuila & 23.0 & 17.3 & 6.6 \\
Nuevo Leon & 14.9 & 6.2 & 3.5 \\
Sonora & 24.4 & 35.6 & 19.6 \\
Tamaulipas & 26.7 & 24.4 & 10.3 \\
Subtotal regional & 21.9 & 19.6 & 9.3 \\
Total nacional & 21.7 & 18.2 & 8.4 \\
& & & \\
\hline
\end{tabular}

FUENTE: Cuadro No, 1 del anexo estad ístico.

CUADRO II. Estados Fronterizos: Estructura del PIB, 1980 (porcentajes).

\begin{tabular}{lrrrrrr}
\hline & $\begin{array}{c}\text { Agrope- } \\
\text { cuario }\end{array}$ & & Minería $\begin{array}{c}\text { Industria } \\
\text { rera }\end{array}$ & $\begin{array}{c}\text { ción } \\
\text { ción }\end{array}$ & $\begin{array}{c}\text { Electri- } \\
\text { cidad }\end{array}$ & $\begin{array}{c}\text { Distribución } \\
\text { y servicios }\end{array}$ \\
\hline Baja California & 9.5 & & 15.6 & 7.2 & 1.5 & 66.2 \\
Coahuila & 6.6 & 11.3 & 22.0 & 7.2 & 0.4 & 52.5 \\
Chihuahua & 14.3 & 6.8 & 17.1 & 3.9 & 1.0 & 56.9 \\
Nuevo León & 3.5 & 3.9 & 36.8 & 4.3 & 0.7 & 50.8 \\
Sonora & 19.6 & 4.7 & 12.1 & 4.2 & 1.7 & 57.7 \\
Tamaulipas & 10.3 & 9.8 & 18.8 & 7.8 & 1.4 & 51.9 \\
Total nacional & 8.9 & 6.8 & 23.0 & 6.5 & 1.0 & 54.3 \\
& & & & & & \\
\hline
\end{tabular}

FUENTE: Cuadro No. 2 del anexo estadístico.

\section{Población económicamente activa.}

Concomitante a una menor participación del sector agropecuario en la actividad económica general, se presenta una reducción en términos absolutos y relativos de la población económicamente activa (PEA) dedicada a las actividades agropecuarias. Sin embargo, todavía la cuarta parte de la población económicamente activa en el año de 1980 tenía su principal fuente de ingresos en el sector agropecuario, lo que constituye un rasgo inequívoco del atraso del país. ${ }^{3}$

\footnotetext{
${ }^{3}$ En paises con la agricultura más desarrollada del mundo como los EUA o Francia, la PEA en las actividades agropecuarias representa entre el 2 y $4 \%$ de la PEA total.
} 
En los estados de la frontera norte, el descenso de la PEA dedicada a las actividades agropecuarias es sistemático y en todos los casos está por debajo del promedio nacional (ver cuadro III).

\section{Trabajos empíricos sobre distribución del ingreso.}

En México,4 este tipo de trabajos constatan que la pobreza de su manifestación más grave ocurre de manera preponderante en el sector rural donde la fuente principal de ingresos es la actividad agrícola.

Con relación a las demás actividades económicas, la agricultura concentra un porcentaje mucho mayor de la población económicamente activa que no recibe ingresos y también un porcentaje más elevado de la PEA en los estratos de ingresos más bajos. Esta estructura de la distribución del ingreso está presente en todos los estados de la frontera norte, con excepción de Chihuahua, donde son los servicios los que aglutinan en los estratos de menor ingreso, el mayor porcentaje de la PEA (ver cuadro IV).

Los argumentos que se han empleado para explicar esta situación, giran en torno a los procesos de transferencia de ingreso del sector campesino al resto de la economía, por medio de tres mecanismos principales: la venta de fuerza de trabajo, la venta de productos y por renta de la tierra.

\section{EL USO DEL SUELO EN LOS ESTADOS FRONTERIZOS.}

El tipo de clima predominante en los estados de la frontera norte, seco y semiseco, 5 determina en buena parte el uso del suelo.

Las cifras del censo de 1970 ponen de manifiesto la preponderancia de las actividades ganaderas en relación a las agrícolas en los estados fronterizos. En conjunto, los seis estados del Norte representan casi la mitad del territorio nacional $(43.7 \%)$, la supcrficie que en esta región se dedica a la ganadería significa un $27.5 \%$ del mismo y la que se considera de labor, sblo el $2.9 \%$ del área total censada en el país.

En la misma zona, la superficic agrícola representa el $6.6 \%$,variando considerablemente de un estado a otro: $3.5 \%$ en Coahuila y $16.6 \%$ en Tamaulipas. En cambio, la ganadería ocupó el $63 \%$ de la superficie total de los estados del norte según el censo de 1970 y se incrementa a un $74.9 \%$ en base a las estimaciones de 1980 . A nivel nacional estas proporciones se inclinan un poco más hacia la agricultura: 23 millones de hectáreas se consideran de la-

\footnotetext{
4Ver por ejemplo: Polı́ticas de consuma y distribución del ingreso. Nora Lustig, subproyecto 10 . SAM, 1980.

5 En un área $\tan$ vasta es inevitable encontrar excepciones importantes, como por ejem plo la Huasteca tamaulipeca y las regiones montañosas de Chihuahua y Sonora.
} 
CUADRO III. Estados Fronterizos: PEA en el sector agropecuario en relación a la PEA total (porcentajes).

\begin{tabular}{lccr}
\hline & $\mathbf{1 9 6 0}$ & $\mathbf{1 9 7 0}$ & $\mathbf{1 9 8 0}$ \\
\hline Baja California & 39.4 & 22.2 & 9.4 \\
Coahuila & 44.8 & 29.6 & 15.7 \\
Chihuahua & 49.8 & 36.4 & 20.8 \\
Nuevo León & 32.2 & 17.3 & 8.3 \\
Sonora & 53.5 & 38.5 & 20.9 \\
Tamaulipas & 50.0 & 33.1 & 18.0 \\
Subtotal regional & 45.0 & 29.1 & 15.3 \\
Total nacional & 54.2 & 39.4 & 25.8 \\
& & & \\
\hline
\end{tabular}

FUENTE: Cuadro No. 3 del anexo estadístico.

CUADRO IV. Estados fronterizos: PEA por rama de actividad y grupos de ingreso mensual. 1980 (porcentajes).

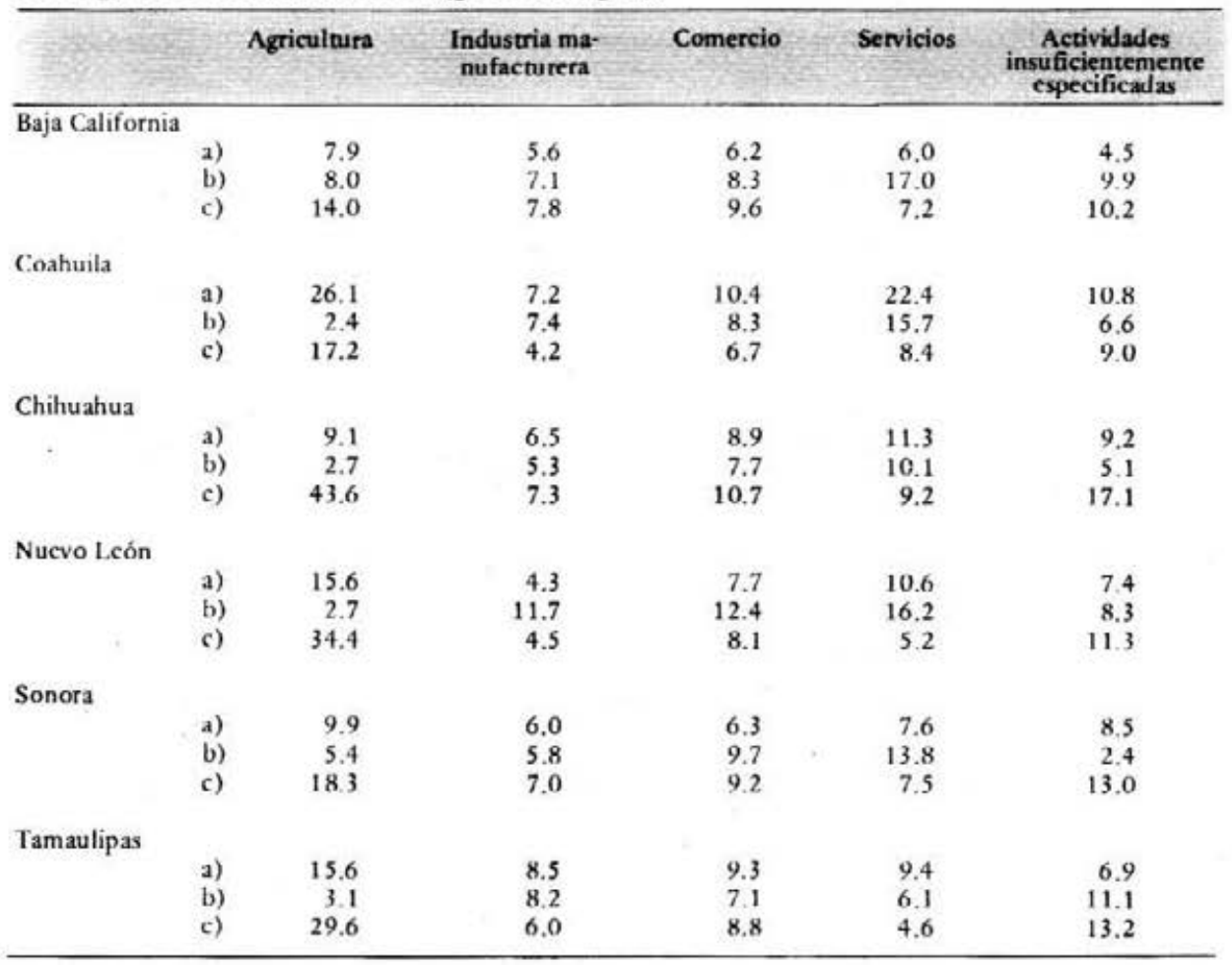

3) Suma de la PEA en los ues estratos inferiores de ingresos (de ) a 1970 pesos).

b) Suma de La PCA en los dos estratos superiores ile ingueson (de 12,111 y mis pesas)

c) No recibieron ingresos.

FUEN TE; $X$ Censo Cenenal de Poblacion y Vivienda, Mexico, 1984. INECG, SPP. 
bor ( $16.5 \%$ del total) en tanto que 74 millones ( $53.3 \%$ de la superficie) tienen un uso ganadero (cuadro V). Se desprende de lo anterior que la ganadería es la actividad más importante en nuestro país, y en especial en los estados del norte, en términos de la superficie que ocupa.

Si consideramos además de la superficie ocupada otros parámetros productivos como son el número de cabezas, el capital invertido y la importancia que ticne en el comercio exterior, la ganadería de vacunos de los estados de la frontera norte ha sido históricamente la de mayor relevancia y la más representativa de la ganadería nacional. 6

Los elementos climatológicos y las condiciones técnico-productivas de los estados de la frontera norte, determinan que el uso del suelo responda -a través de una ganadería de vacunos de carne de tipo extensivo que erosiona los agostadcros por sobrepastoreo- a la integración productiva de la región norte de México con el sur de los EUA.

Se ha dicho de esta ganadería "que no reconoció fronteras rígidas con el sudoeste de los Estados Unidos, conformando una estructura muy cstable de explotación. .. entrelazada y dependiente de los ciclos ganaderos en ese país"?

La ganadería de los estados de la frontera norte está organizada productivamente en torno a la exportación de becerros en pie, la cual representó en promedio entre 1960 y 1980 más del $70 \%$ de la producción anual de becerros y en algunos años de alta demanda, la totalidad de la producción regional. ${ }^{8}$

No deja de ser revelador el hecho de que el empleo que se da a $45.8 \mathrm{mi}-$ llones de hectáreas en los estados del Norte ${ }^{9}$, está en función de los requerimientos de la ganadería norteamericana, la cual completa su producción con la importación de becerros de Canadá, México y Centroamérica.

La ganadería no sólo ocupa la mayor parte de la superficie en cada uno de los estados del Norte, sino que es además el subsector más dinámico: en Sonora el valor de la producción pecuaria ${ }^{10}$ se eleva a una tasa promedio de $10.0 \%$ anual en el periodo 1970-80 en tanto que el agrícola sólo crece 1.0\% .

\footnotetext{
${ }^{6}$ Sin embargo, es importante señalar que en los últimos veinte años el dinamismo mayor se encuen tra en las zonas tropicales y subtropicales. Ver: Las ramificaciones internacionales de la inchistria del ganado vacuno en México. Dr. Erncst Feder, CODAI, SARH, 1982.

71: sistema ganadero-industrial: su estructura y desarrollo 1960-80. Dr. Nicolás Reig. Documento de Trabajo para el Desarrollo Agroindustrial. No. 8, CODAI, SARH, 1982, p. 124.

${ }^{8}$ Nicolás Reig, op. cit, , p, 135

${ }^{9}$ En Sonora se estima que el agostadero es de 15 millones de hectáreas y en Chihuahua de 18,7 millo. nes. Planes de Desarrollo Agropecuario y Forestal, 1982-88. SARH.

10 Precios constantes de 1960.
} 
CUADRO V. Estados fronterizos: uso del suelo (miles de hectáreas). 1970 y 1980.

\begin{tabular}{|c|c|c|c|c|c|}
\hline & $\begin{array}{l}\text { Sup. total } \\
\text { censada }\end{array}$ & Agricola & $\begin{array}{c}\text { Ganadera } \\
1970(1)\end{array}$ & $\begin{array}{c}\text { Ganadería de } \\
\text { vacunos } \\
1980(2)\end{array}$ & Otras (3) \\
\hline Baja California & 2,732 & 324 & 1,135 & $(2,393)$ & 1,273 \\
\hline Sonora & 12,847 & 813 & 10,167 & $(12,031)$ & 1,867 \\
\hline Chihuahua & 21,263 & 1,068 & 15,989 & $(15,837)$ & 4,206 \\
\hline Coahuila & 13,265 & 470 & 5.199 & $(8,315)$ & 7,576 \\
\hline Nuevo León & 4,637 & 323 & 3,022 & $(2,961)$ & 1,292 \\
\hline Tamaulipas & 6,486 & 1,074 & 3,035 & $(4,037)$ & 2,377 \\
\hline Subtotal & 61,230 & 4,072 & 38,547 & $(45,844)$ & 18,611 \\
\hline Total nacional & 139,868 & 23,138 & 74,499 & $(78,005)$ & 42,231 \\
\hline \multicolumn{6}{|c|}{ Porcentajes } \\
\hline Baja California & 100.0 & 11.9 & 41.5 & $(87.5)$ & 46.6 \\
\hline Sonora & 100.0 & 6.3 & 79.1 & $(93.6)$ & 14.6 \\
\hline Chihuahua & 1000 & 5.0 & 75.2 & $(74.5)$ & 19.8 \\
\hline Coahuila & 100.0 & 3.5 & 39.2 & $(62,7)$ & 57.3 \\
\hline Nucvo Lcón & 100.0 & 7.0 & 65.2 & (63.9) & 27,8 \\
\hline Tamaulipas & 100.0 & 16.6 & 46.8 & $(66.4)$ & 36,6 \\
\hline Subtotal & 100.0 & 6.6 & 63.0 & $(74,9)$ & 30.4 \\
\hline Total nacional & 100.0 & 16.5 & 53.3 & $(57.7)$ & 30.2 \\
\hline \multicolumn{6}{|c|}{ Porcentajes } \\
\hline Baja California & 1.9 & 1.4 & 1.5 & 3.1 & 3.0 \\
\hline Sonora & 9.2 & 3.5 & 13.6 & 15,4 & 4.4 \\
\hline Chihuahua & 15.2 & 4.6 & 21.4 & 20.3 & 10.0 \\
\hline Coahuila & 9.5 & 2.0 & 7.0 & 10.7 & 18.0 \\
\hline Nucvo León & 3.3 & 1.4 & 4.1 & 3.8 & 3.1 \\
\hline Tamaulipas & 4.6 & 4.7 & 4.1 & 5.5 & 5.6 \\
\hline Subtotal & 43.7 & 17.6 & 51.7 & 58.8 & 44.1 \\
\hline Total nacional & 100.0 & 100.0 & 100.0 & 100,0 & 100,0 \\
\hline
\end{tabular}

(1) Con pastos naturales en cerros $y$ llanuras.

(2) Estimación. Nicolás Reig, 1980 ,

(3) Incluye: Bosques, inculta prodactiva y no aptas ni para ageicultura ni ganadería.

FUENTE: $V$ Censo Agricola Candero y Ejidd, 1970.

En Nuevo León la ganadería representó el $48.1 \%$ del PIB en 1960 y aumenta su participación al $65.2 \%$ en el quinquenio 1976-1980; su tasa de crecimiento en el periodo $1960-1980$ fue de $4.0 \%$, mientras que la del subsector agrícola de $0.4 \%$. Esta tendencia se presenta también en Baja California, aunque en forma más marcada: el subsector agrícola se retrae a un ritmo de $-5.7 \%$ y el pecuario se expande $18.3 \%$ anual en el periodo 1962-70. Sólo en el estado de Chihuahua se presenta un proceso inverso; en el lapso 1964-78 el subsector agrícola aumenta más rápidamente que el pecuario, no obstante, éste sigue siendo el principal generador de divisas en el estado, 11

Resulta paradójico que la actividad más importante y de mayor dinamismo es también la que menores recursos de inversión ha recibido por parte

\footnotetext{
${ }^{11}$ No se pudo obtener información para los estados de Coahuila y Tamaulipas. Ver: Planes de Desarrollo Agropecuario y Forestal, 1982-88. SARH.
} 
del gobierno. El carácter estructural de la ganadería de los estados del Norte: extensividad, baja productividad, integración productiva con los EUA, su concentración en pocas manos y su reducida generación de empleos, pone en tela de juicio la racionalidad del uso de casi 50 millones de hectáreas que a ella se dedican.

\section{LA AGRICULTURA EN LA FRONTERA NORTE.}

\section{El marco global.}

La crisis del sector agrícola que empieza a manifestarse en la segunda mitad de la década de los sesentas con un muy lento crecimiento de la producción y con un retraimiento de la superficie cosechada, se expresa plenamente en los primeros años de los setentas con las significativas importaciones de maíz, trigo, sorgo y soya. Su permanencia hasta nuestros días es un hecho por todos reconocido.

Entre las manifestaciones más importantes de esta crisis está, desde luego, la pérdida de au tosu ficiencia en la producción de granos básicos, ${ }^{12}$ la presencia por primera vez en 1980 , de saldos negativos en la balanza agropecuaria, el deterioro de la dieta de la población rural y de los grupos urbanos de más bajos ingresos y la violencia en cl campo.

Existen tres factores que inciden directamente en la problemática señalada: en promedio, la superficie cosechada por trabajador ocupado en la agricultura ha decrecido; ${ }^{13}$ las tasas de crecimiento de los rendimientos promedio también han disminuido y por último, ha habido un cambio notable en la composición de cultivos, o sea, que la orientación de la agricultura se ha modificado.

Presentado en forma un poco simplista, podemos decir que los dos primeros elementos están asociados a las hipótesis que sostienen que la crisis agrícola tiene su origen en el papel subordinado del sector agrícola del resto de la economía y en especial en relación con el sector industrial y del papel que juegan en este esquema las transferencias inter e intrasectoriales, que algunos autores inclusive llevan a nivel internacional. ${ }^{14}$

El cambio en la orientación de la agricultura ha sido explicado por varios autores ${ }^{15}$ como un resultado del proceso de internacionalización de la

\footnotetext{
$12 \mathrm{El}$ paŕs nunca fue autosuficiente en la producción de cultivos para el consumo animal.

${ }^{13}$ Existe una abundante literatura con relación a estos planteamien tos y una in teresante polémica acer. ca de los mismos. Para citar sólo algunos: Gómez Oliver (1977), Michael Gutelman (1971), Alan
Janvry (1975).

14Ver: Barkin (1982), Rama (1977), Rama y Rello (1977). 15 La superficie cosechada total a partir de 1965 crece a tasas muy bajas e incluso negativas en algunos
periodos.
} 
agricultura. Según esta tesis, las transformaciones de la estructura productiva del agro tienen una estrecha relación con los requerimientos del capital internacional y son resultado del papel que se asigna a la agricultura mexicana dentro de los esquemas de la división internacional del trabajo.

Dentro de esta visión los dos grandes cambios en la orientación de la agricultura estaban representados: 1) por el carácter agroexportador del sistema frutas y legumbres y de algunos productos ganaderos y, 2) por lo que se ha denominado el proceso de ganaderización de la agricultura.

La evolución reciente de la ganadería y el análisis detallado de los cambios en el patrón de cultivos a nivel nacional, regional y estatal, ${ }^{16}$ echaron por tierra la multicitada vocación agroexportadora de la agricultura y colocaron en un contexto diferente el problema de la ganaderización. Demos algunos elementos en este sentido:

1) Los problemas estructurales de la ganadería reflejados en una menor oferta de carne de bovinos, frente a un mercado interno creciente, por lo menos hasta el año de 1982, dieron lugar a la cancelación de las exportaciones de las distintas carnes - cortes, canales y carne deshuesada- hacia fines de los setentas con el objeto de poder cubrir el mercado interno. A partir de 1985, y no obstante el descenso del consumo originado por la crisis, se efectúan las primeras importaciones de ganado en pie y carne en canal de Centroamérica.

2) En términos del uso del suelo, la agricultura mexicana está cada vez menos volcada hacia la exportación. Tomando en cuenta los ocho principales productos de exportación ${ }^{17}$ vemos que el área dedicada a su cultivo se reduce del $13.7 \%$ en 1960 al $8.3 \%$ en 1979 . Si consideramos la superficie cosechada con un grupo de cultivos que hemos denominado de consumo directo, ${ }^{18}$ entre los cuales se encuentran algunas hortalizas que en parte se exportan y en parte satisfacen el consumo interno, observamos que su área bajo cultivo se ha mantenido estable en un período de 19 años, representando el $2.1 \%$ de la superficie cosechada nacional en 1960 y en 1979.

Consideramos que la exportación difícilmente puede ser el objetivo y la vocación de la agricultura mexicana por las siguientes razones: ${ }^{19}$

2.1.) La agricultura de exportación está siendo sustituida por una agricultura cuyo objetivo es el mercado interno, sobre todo aquel de ingresos

\footnotetext{
${ }^{16}$ Ver: Competencia entre agricultura y ganader źa por el uso de la tierra en México, 1960-79. Rosario Pérez Espejo. (En proceso de publicación por la UNAM).

${ }^{17}$ Algodón, café, tabaco, henequén, fresa, melón, jitomate y garbanzo.

${ }^{18}$ Cacahuate, papa, cebolla, chícharo, chile verde, chile seco, camote, sandŕa.

${ }^{19}$ El comercio de productos agropecuarios en tre México y los EUA. Rosario Pérez Espejo. Proyecto de Investigación presentado al IIEc, UNAM, enero de 1984 (66 pp).
} 
medios y altos. Por otra parte, se ha demostrado que cualquier proceso redistributivo -improbable bajo las actuales reglas de política económicapor pequeño que sea, provocará un incremento considerable en la demanda de productos alimenticios.

2.2.) El mercado internacional de algunos productos tropicales no se muestra favorable ya que existe una fuerte competencia por parte de otros países productores, $y$ un férreo control del mismo por los países industrializados.

2.3.) Los costos de producción de los productos agrícolas son cada vez más altos debido al componente de insumos importados que forman parte del mismo. En este sentido, el esquema agroexportador tiende a consumir más divisas de las que genera.

3) En cuanto al proceso de ganaderización, estamos totalmente de acuerdo en que éste se presenta, y que además, es muy importante. En lo que no estamos de acuerdo es en considerar que la expansión de las distintas ganaderías y de la agricultura estén determinadas por los fenómenos de internacionalización de la agricultura.

Desde nuestro punto de vista, la ganaderización de la agricultura o la competencia por la tierra entre agricultura y ganadería, asume dos modalidades: una de carácter directo y otra indirecta. La competencia directa es la relativa a la ocupación que agricultura y ganadería hacen de una determinada porción del territorio, como actividades económicas principales. ${ }^{20} \mathrm{La}$ competencia indirecta es el empleo de extensiones cada vez mayores de la superficie

agrícola bajo cultivo, con fines de alimentación animal. En este caso se trata de una subordinación agrícola representada por la expansión de cultivos que en forma directa o como componentes de los alimentos balanceados van a ser consumidos por las distintas ganaderías. A nivel nacional se encontró que de 1950 a 1960 tanto la agricultura como la ganadería se expandieron con base en las superficies clasificadas como inexplotacas e incxplotables, las cuales se reducen de $22 \%$ en 1950 a $4 \%$ en 1970 . Sin embargo, en la siguiente década la ganadería crece en términos relativos y absolutos en tanto que la superficie censada como agrícola se reduce en 14 millones de hectáreas (cuadro VI).

La superficie de labor en 1970 se estimó en 23 millones de hectáreas y la inculta productiva ${ }^{21}$ en 7; total: 30 millones de hectáreas. ¿Qué uso ten-

\footnotetext{
${ }^{20}$ Según la clasificación censal, la actividad económica principal es aquella que origina el $60 \%$ o más de las ventas de la unidades de producción.

21 Superficie donde se realizan labores de recolección de productos no cultivados como lechuguilla, ixtle, barbasco, candelilla, etc.
} 
drían los restantes 33 millones de los 63 considerados como agrícolas por el censo?.

En relación con la competencia indirecta, tenemos que en 1960 los cultivos que tenían un uso en la alimentación animal ${ }^{22}$ representaron el $3.1 \%$ de la superficie cosechada y en 1979 su participación había ascendido al $23.3 \%$. Su crecimiento promedio anual fue de $12.7 \%$ en ese periodo. La contraparte fue un descenso en la ponderación de los cultivos básicos del $69.5 \%$ en 1960 al $50.1 \%$ en 1979 , y en los cultivos de exportación del $13.7 \%$ al $8.3 \%$ en los mismos años.

CUADRO VI. Superficie agrícola y ganadera (millones de hectáreas).

\begin{tabular}{lcccc}
\hline & 1950 & 1960 & 1970 & 1980 \\
\hline Total agrícola & 47.5 & 76.7 & 63.0 & \\
Total ganadera* & 50.2 & 55.5 & 65.8 & 77.6 \\
Total ganadera* & 47.9 & 50.3 & 54.3 & \\
\hline
\end{tabular}

- Estimaciones del Dr. Nicolás Reig. El sistema ganadero-industrial: su estructura y desarrollo 1960 1980.

\section{Características generales de la agricultura fronteriza.}

La superficie ocupada por la agricultura en los estados del Norte es de 4 millones de hectáreas y representa el $6.5 \%$ del área censada en la región. Sin embargo, su ponderación en relación con la superficie cosechada nacional es mucho mayor, representando en 1983 el $19.2 \%$ de la misma. A este monto contribuyen de manera muy desigual cada uno de los estados de la frontera: Tamaulipas, Chihuahua y Sonora aglutinan el $85 \%$ de la superficie cosechada en la región y el $76 \%$ de la superficie de riego. En estos estados se genera el $77 \%$ del valor de la cosecha (cuadro VII). Es indudable que el ricgo ha sido un elemento básico para el desarrollo de la agricultura cn los estados de la frontera norte. Con la excepción de Tamaulipas, las condiciones climatológicas de las áreas no montañosas de los demás estados imponen su uso como una necesidad; pero son factores de tipo histórico y político los que han conducido a privilegiar a algunos estados en relación con otros. El gran aporte que constituye el riego se comprueba en lo siguiente: en Sonora, por ejemplo, el $98 \%$ de la superficie cosechada cuenta con riego, representando el $17 \%$ de la superficie cosechada regional; el valor de la cosecha es proporcionalmente mucho mayor, alcanzando el $28.3 \%$ del total regional. En Tamauli-

22 Incluimos sorgo, distintos forrajes y también al cártamo y la soya, pues su dinamismo es resultado be la demanda de pastas y uso de aceites. 
CUADRO VII. Estados fronterizos: superficie y valor de la cosecha.

1981

\begin{tabular}{|c|c|c|c|c|c|c|c|c|c|c|c|c|}
\hline & & SUI & PERFICIE & cosec & HADA & & & VALOR & DE LA P & RODUC & CCION & \\
\hline & $\begin{array}{l}\text { RIEC } \\
\text { Miles } \\
\text { de Has. }\end{array}$ & $\begin{array}{l}\text { GO } \\
\text { 8. } \%\end{array}$ & $\begin{array}{l}\text { TEMP } \\
\text { Miles } \\
\text { de Has. }\end{array}$ & $\begin{array}{r}\text { ORAL } \\
\%\end{array}$ & $\begin{array}{l}\text { TOT } \\
\text { Miles } \\
\text { de Has. }\end{array}$ & TAL & $\begin{array}{r}\text { RIE } \\
\text { Millones } \\
\text { de pesos }\end{array}$ & & $\begin{array}{l}\text { TEMPO } \\
\text { Millones } \\
\text { de pesos }\end{array}$ & $\begin{array}{r}\text { RAL } \\
\%\end{array}$ & $\begin{array}{r}\text { TOT } \\
\text { Millones } \\
\text { de pesos }\end{array}$ & AL \\
\hline Baja California & 193 & 94.2 & 12 & 5.8 & 205 & 100.0 & 6,876 & 99.6 & 38 & 0.5 & 6,914 & 100.0 \\
\hline oal & 167 & 74.6 & 57 & 25 & 224 & 100.0 & & 9 & 353 & 5.4 & 88 & 100.0 \\
\hline Chihuahua & 291 & 26.0 & 830 & 74.0 & 1,121 & 100.0 & 9,073 & 58.2 & 6,529 & 41.8 & 15,602 & 100.0 \\
\hline Nuevo León & 128 & 51.8 & 119 & 48.2 & 247 & 100.0 & 3,371 & 72.9 & 1,252 & 27.1 & 4,623 & 100.0 \\
\hline Sonora & 754 & 97.6 & 19 & 2.4 & 773 & 100.0 & 22,141 & 99.5 & 114 & 0.5 & 22,255 & 100.0 \\
\hline Tamaulipas & 496 & 25.6 & 1,140 & 74.4 & 1,936 & 100.0 & 9,468 & 41.4 & 13,384 & 58.6 & 22,852 & 100.0 \\
\hline SUB TO'TAL & 2,029 & 45.0 & 2,477 & 55.0 & 4,506 & 100.0 & 57,064 & 72.5 & 21,670 & 27.5 & 78,734 & 100.0 \\
\hline TOTAL NACIONAL & 5.133 & 24.7 & 15,623 & 75.3 & 20,756 & 100.0 & 169,504 & 45.3 & 205,183 & 54.7 & 374,687 & 100.0 \\
\hline & & & & 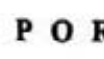 & & & & & & & & \\
\hline Baja & 3.8 & 9.5 & 0.1 & 0.5 & 10 & 4.5 & 4.1 & 12.0 & & 02 & 1.8 & 8.8 \\
\hline & 3.2 & & & 2 & & 5 & 3 & 1 & 0.2 & 7 & 1.7 & 8.2 \\
\hline ua & 5.7 & 14.3 & 5.3 & 33.5 & 5.4 & 24.9 & 5.4 & 15.9 & 3.2 & 30.1 & 4.2 & 19.8 \\
\hline Nuevo León & 2.5 & 6.3 & 0.8 & 4.8 & 1.2 & 5.5 & 2.0 & 5.9 & 0.6 & 5.8 & 1.3 & 5.9 \\
\hline רחק & 14.7 & 37.2 & 0.1 & 0.8 & 3.7 & 17.2 & 13.1 & 38.9 & 0.1 & 0 & 5.9 & 28.3 \\
\hline $\mathrm{Ta}$ & 9.6 & 24.5 & 9.2 & 58.1 & 9.3 & 42.9 & 5.5 & 16.6 & 6.5 & 61.8 & 6.1 & 29.0 \\
\hline SUB TOTAL & 39.5 & 100.0 & 15.9 & 100.0 & 21.7 & 100.0 & $33: 7$ & 100.0 & 10.6 & 100.0 & 21.0 & 100.0 \\
\hline TOTAL NACIONAL & 100.0 & & 100.0 & & 100.0 & & 100.0 & & 100.0 & & 100.0 & \\
\hline
\end{tabular}

FUENTE: Anuario estadístco. Producción Agricola Nactonal, i981, SARH-DGEA. 
pas, en cambio, el $25 \%$ de la superficie es irrigada; en el estado se encuentra el $43 \%$ de la superficie cosechada regional pero en ella sólo se genera el $29 \%$ del valor de la cosecha. Es evidente que el riego ha permitido primero, la práctica de una agricultura más tecnificada, con rendimientos altos para los niveles nacionales, pero que depende de un paquete de insumos que cada vez son más caros y en cuya producción el país no es autosu ficiente; este modelo, por otra parte, parece haber llegado al límite de su expansión; y segundo, la producción de cultivos de mayor valor económico.

\section{Participación a nivel nacional.}

La agricultura de los estados de la frontera norte, medida en términos de superficies cosechadas, ha representado en un periodo que va de 1960 a 1983, más o menos la quinta parte de la agricultura mexicana (cuadro VIII). Sin embargo, a nivel de determinados cultivos su participación ha sido y es fundamental.

En orden de importancia encontramos que en esta región y básicamente en Chihuahua, el $84 \%$ de la superficie total ocupada se cosecha con avena en grano; no obstante su secular declive en la región, en 1983 el $76 \%$ de la superficie cosechada de algodón se encontraba en los estados de la frontera norte; las áreas trigueras de esta región representaron el $60 \%$ de la superficie total dedicada a este cultivo. A pesar de los elevados rendimientos que se obrienen, en especial en Sonora y Baja California, fruto sin duda de la revolución verde 23 y de que nuestro país exporta semilla mejorada de trigo, la producción no logra cubrir el mercado interno. La superficie cosechada con soya y cártamo -cultivos relativamente recientes en nuestro país - representa en ambos casos el $46 \%$ de la superficie nacional. A partir de 1971 se empieza a discriminar en la estadística agrícola el destino de ciertos cultivos; así vemos que el grueso de la superficie que antes aparecía cultivada con garbanzo para consumo humano, en 1971 se registra como garbanzo forrajero.

El conjunto de cultivos forrajeros, garbanzo, cebada, avena, sorgo y maíz, tiene elevadas tasas de crecimiento en todo el país y en los estados de la frontera norte, donde se encuentran importantes extensiones cultivadas con estos forrajes: el $\mathbf{5 7 \%}$ de la superficie con sorgo forrajero se localiza allí, el $50 \%$ de la cebada forrajera, $51 \%$ de avena forrajera, $40 \%$ de sorgo y $33 \%$ de alfalfa.

De los cultivos considerados de exportación, ${ }^{24}$ los estados de la frontera fueron importantes productores de algodón cuya superficie en términos

\footnotetext{
${ }^{23}$ Los rendimientos de trigo en México son mucho más al tos que en los EUA, pero el trigo en nuestro pasis se siembra bajo riego, mientras que en los EUA es de temporal.

24Ver apartado sobre metodología del trabajo. Rosario Pérez Espejo, op. cit.
} 
absolutos y relativos se reduce sistemáticamente de 1960 a 1977 . En el primer año se cosechaban en la región 758 mil hectáreas que representaban el $84 \%$ de la superficie nacional. Para 1977, la superficie se habra reducido a 252 mil hectáreas en la región, cuya ponderación, debido a una relocalización del cultivo hacia el Sur - Chiapas y Michoacán especialmente- se redujo al $56.6 \%$ de la superficie nacional. En 1983 la superficie cosechada se redujo aún más ocupando 177 mil hectáreas aunque su peso relativo en la región aumenta al $76 \%$.

Con excepción del trigo, los estados de la frontera norte nunca han sido importantes productores de cultivos básicos. La superficie cosechada con arroz es insignificante; la extensión ocupada con maíz ha oscilado entre un mínimo de $6.9 \%$ en 1971 y un máximo de $11.6 \%$ en 1977 , en relación al total nacional. En frijol la participación fluctuó entre un mínimo de $7.4 \%$ en 1977 y un máximo de $14.1 \%$ de la superficie cosechada total (Cuadro 4 del anexo).

\section{Orientación del patrón de cultivos.}

Una mirada al cuadro 11 del anexo nos revela lo siguiente:

1o. El único cultivo importante dentro de los de exportación es el algodón, pero si en algún lado el modelo agroexportador se ha desplomado, es precisamente en los estados de la frontera norte. Las causas de este proceso han sido internas (presencia de plagas en Tamaulipas por ejemplo) pero también externas: problemas de precios internacionales, competitividad, etc. La ponderación de los cultivos de exportación en el total se reduce del $34.5 \%$ en 1960 al $5.4 \%$ en 1983 , presentándose este proceso en todos los estados de la frontera.

2o. La segunda modificación de importancia es la expansión de las áreas cosechadas con cultivos para consumo animal, las cuales incrementan su participación de un $2.8 \%$ en 1960 al $33.9 \%$ en 1983 . El proceso de ganaderización indirecta en los estados del Norte y en el resto del país obedece, desde nuestro punto de vista, a los requerimientos del mercado interno, vía las demandas de la porcicultura y la avicultura. El enorme dinamismo de estas dos ganaderías se debe fundamentalmente a los cambios en los sistemas de alimentación, en los cuales el sorgo juega un papel muy importante. La expansión de este cultivo ha permitido los incrementos en la producción y en los consumos per cápita de carne de cerdo, de ave y de hucvo. En la década de los cincuentas el país no importaba sorgo, pero importaba hucvo y alimentos balanceados, además de algunos básicos.

No en todos los estados de la frontera el aumento de los cultivos de consumo animal se da por la expansión del sorgo. Esto sucede así, en Tamau- 
lipas y Nuevo León; en Baja California y Coahuila se incrementa la superficie cosechada con alfalfa y en Chihuahua con avena forrajera. En ambos casos se atiende al ganado lechero. La paradoja se presenta en Sonora, estado que cuenta con una porcicultura y una avicultura sumamente dinámicas, pero donde casi no se produce sorgo. 25

3o. La superficie cosechada con cultivos básicos sufre una notable reducción en su participación porcentual, sobre todo si sc toma como referencia 1965, año de mayor expansión agrícola. La ponderación de básicos disminuye del $56.4 \%$ en 1965 al $39.4 \%$ en 1983 . El proceso de reducción relativa en los alimentos básicos en el patrón de cultivos, rige para todos los estados, con excepción de Baja California. En el estado de Tamaulipas que posee las áreas sorgueras y maiceras más importantes en la región, se estableció una fuerte competencia entre ambos cultivos por las áreas bajo riego. A partir de 1971 y con algunos años como excepción la superficie cosechada con sorgo ha sido may or que la cosechada con maíz.

4o. Por último, hay que destacar el incremento sistemático tanto en términos absolutos como en términos relativos de la superficie cosechada con cártamo y soya hasta el año de 1979. En la última observación -1983-esta tendencia se revierte en Sonora y Tamaulipas, que son los estados que aglutinan las superficies más importantes cosechadas con estos cultivos. Aunque la transformación de estas oleaginosas ${ }^{26}$ en aceites y pastas esté, en buena medida, en manos de empresas transnacionales, sin embargo, su finalidad es la satisfacción de la demanda interna, ya sea como aceites comestibles o como pastas para la elaboración de alimentos balanceados.

Estos son los cambios más relevantes en la orientación de la agricultura del conjunto de los estados de la frontera norte. Cada uno de ellos tiene sus especificidades pero las tendencias de tipo general se presentan en todos.

El elemento determinante de estos cambios cs, a nuestro juicio, el mercado interno, el cual se ha modificado sustancialmente en los últimos años por el crecimiento de la población y el proceso de urbanización.

\footnotetext{
25 Hasta principios de 1985 existía un acuerdo entre CONASUPO y el estado de Sonora mediante el zual la primera proporcionaba sorgo de importación subsidiado y la segunda proporcionaba trigo a los - olinos del país.

:6 La soya es una leguminosa pero dadas sus posiblidades acciteras, se le ubica con frecuencia dentro $\because$ Las oleaginosas.
} 


\section{BIBLIOGRAFIA}

BARKIN, David. El uso de la tierra agrícola en México. Ponencia presentada al 1er. Seminario de Economía Agrícola del Tercer Mundo. IIEc, UNAM, julio 1982 .

DE HANVRY, Alain. The political economy of rural development in Latinoamerica: an interpretation. American Journal of Agricultural Economics, vol. 57, No. 3, agosto 1975 .

DELGadillo, M. Javier. Análisis espacial de la frontera norte de México. Tesis, Facultad de Filosofía y Letras, UNAM, 1982.

FEDER, Ernest. Las ramificaciones internacionales de la industria del ganado vacuno en México. Documento de trabajo para el Desarrollo Agroindustrial, No. 8, CODAI, SAR, 1982.

GOMEZ, A. Luis, Hacia una fundamentación análitica para una nueva estrategia de desarrollo. CIDER, diciembre, 1977.

GUTELMAN, Michel. Capitalismo y reforma agraria en México, ERA, 1971.

PEREZ E. Rosario. Competencia en tre agricultura y ganadería por el uso de la tierra en México: 1960-79. (En proceso de publicación UNAM).

El comercio de productos agropecuarios entre México y los EUA. Proyecto de investigación presentado al IIEc, UNAM, enero 1984.

RAMA, Ruth. "Empresas trasnacionales y agricultura mexicana: el caso de las procesadoras de frutas y legumbres", en Investigación Económica, año 37 , vol. 37 , no. 143 .

RAMA, Ruth y RELLO, Fernando."La agroindustria mexicana: su articulación con el mercado mundial", en Investigación Económica, enero-marzo 1979 , no. 147 , vol. 38 .

REIG, Nicolás. El sistema ganadero-industrial: su estructura y desarrollo, 1960-80. Documento de Trabajo para el Desarrollo Agroindustrial, No. 8. CODAI, SARH, 1982.

SECRETARIA DE AGRICULTURA Y RECURSOS HIDRAULICOS, Planes de Desarrollo Agropecuario y Forestal 1982-88.

SECRETARIA DE PROGRAMACION Y PRESUPUESTO. Sistema de Cuentas Nacionales de México. Producto intemo bruto por entidad federativa, 1980.

XIRAU, Joaquín y Miguel Díaz. Nuestra dependencia fronteriza. F.C.E., México, 1976. 


\section{ANEXO ESTADISTICO}

CUADRO 1. Estados fronterizos: PIB total 1950, 1960, 1970 y 1980. ( 1950 y 1960 millones de pesos de $1950 ; 1970$ y 1980 , millones de pesos).

\begin{tabular}{lrrrr}
\hline & $\mathbf{1 9 5 0}$ & $\mathbf{1 9 6 0}$ & $\mathbf{1 9 7 0}$ & $\mathbf{1 9 8 0}$ \\
\hline Baja California & $1,039.34$ & $2,028.57$ & $95,859.8$ \\
Coahuila & $1,479.24$ & $2,372.89$ & $126,360.7$ \\
Chihuahua & $1,902.54$ & $3,204.40$ & $120,438.8$ \\
Nucvo León & $1,855.01$ & $4,733.87$ & $250,772.1$ \\
Sonora & $1,269.69$ & $2,284.77$ & $104,906.3$ \\
Tamaulipas & $1,465.97$ & $2,053.09$ & $148,859.8$ \\
SUBTOTAL REGIONAL & $9,011.73$ & $16,677.59$ & $847,197.5$ \\
TOTAL NACIONAL & $41,060.12$ & $74,215.18$ & $4 ' 276,490.4$
\end{tabular}

PIB, sector agropecuario

\begin{tabular}{lrrrr}
\hline & $\mathbf{1 9 5 0}$ & $\mathbf{1 9 6 0}$ & $\mathbf{1 9 7 0}$ & \multicolumn{1}{c}{$\mathbf{1 9 8 0}$} \\
\hline Baja California & 223.45 & 353.34 & & $9,076.8$ \\
Coahuila & 340.06 & 411.56 & $8,344.9$ \\
Chihuahua & 437.98 & 893.51 & $17,273.6$ \\
Nuevo León & 275.96 & 295.13 & $8,831.4$ \\
Sonora & 309.79 & 812.28 & $20,579.5$ \\
Tamaulipas & 391.69 & 502.26 & $15,258.5$ \\
SUBTOTAL REGIONAL & $1,978.93$ & $3,268.08$ & $79,564.7$ \\
TOTAL NACIONAL & $8,902.00$ & $13,538.00$ & & $357,131.1$ \\
\hline
\end{tabular}

FUENTES: 1950, 1960: Producto Interno Bruto por entidad federativa: 1900, 1940, 1950, 1960. Kirsten A. Appendini CEED. COLMEX (mimeo). 1970:

1980: Cuentas Nacionales. PIB por entidad federativa. INEGI. SPP. 
CUADRO 2. Estados fronterizos: PIB por sector de actividad, 1980 (millones de pesos).

\begin{tabular}{lrrrrrr}
\hline & $\begin{array}{c}\text { Baja } \\
\text { California }\end{array}$ & Coahuila & Chihuahua & Nuevo León & Sonora & Tamaulipas \\
\hline 1. Agropecuario & $9,076.8$ & $8,344.9$ & $17,273.6$ & $8,831.4$ & $20,579.5$ & $15,258.5$ \\
2. Minería & 19.7 & $14,300.6$ & $8,171.7$ & $9,689.8$ & $4,956.8$ & $14,595.1$ \\
3. Industria manufacturcra & $14,935.2$ & $27,755.2$ & $20,616.9$ & $92,204.2$ & $12,698.8$ & $28,118.2$ \\
4. Construcción & $6,932.4$ & $9,169.6$ & $4,695.3$ & $10,661.0$ & $4,446.7$ & $11,600.1$ \\
5. Electricidad & $1,410.8$ & 459.1 & $1,234.7$ & $1,678.4$ & $1,751.4$ & $2,049.1$ \\
6. Distribución & $36,827.5$ & $36,300.7$ & $41,665.4$ & $71,651.2$ & $35,523.1$ & $44,197.8$ \\
7. Resto de servicios & $26,657.4$ & $30,030.6$ & $26,781.2$ & $56,056.1$ & $24,950.0$ & $33,041.0$ \\
TOTAL & $95,859.8$ & $126,360.7$ & $120,438.8$ & $250,772.1$ & $104,906.3$ & $148,859.8$ \\
\hline
\end{tabular}

FUENTE: Cuentas nacionales. PIB por entidad federativa, 1980, INEGI. SPP. 
CUADRO 3. Estados fronterizos: PEA en el sector agropecuario y PEA total: 1960, 1970, 1980 (miles de personas).

\begin{tabular}{lrrrrrr}
\hline & \multicolumn{1}{c}{$\begin{array}{c}\text { PEA EN EL SECTOR } \\
\text { AGROPECUARO }\end{array}$} & \multicolumn{4}{c}{ PEA TOTAL } \\
& $\mathbf{1 9 6 0}$ & $\mathbf{1 9 7 0}$ & $\mathbf{1 9 8 0}$ & $\mathbf{1 9 6 0}$ & $\mathbf{1 9 7 0}$ & $\mathbf{1 9 8 0}$ \\
\hline Baja California & 66 & 49 & 38 & 167 & 222 & 403 \\
Coahuila & 129 & 86 & 76 & 288 & 289 & 483 \\
Chihuahua & 188 & 151 & 138 & 376 & 416 & 665 \\
Nuevo León & 134 & 109 & 101 & 251 & 284 & 484 \\
Sonora & 117 & 85 & 67 & 363 & 491 & 804 \\
Tamaulipas & 167 & 126 & 112 & 334 & 381 & 624 \\
SUB TOTAL REGIONAL & 801 & 606 & 532 & 1,780 & 2,085 & 3,463 \\
TOTAL NACIONAL & 6,145 & 5,103 & 5,700 & 11,332 & 12,955 & 22,066 \\
\hline
\end{tabular}

FUENTE: Censos de Población y Vivienda: 1960, 1970, 1980. 
CUADRO 4. Estados fronterizos: participación en el total por cultivo (porcentajes).

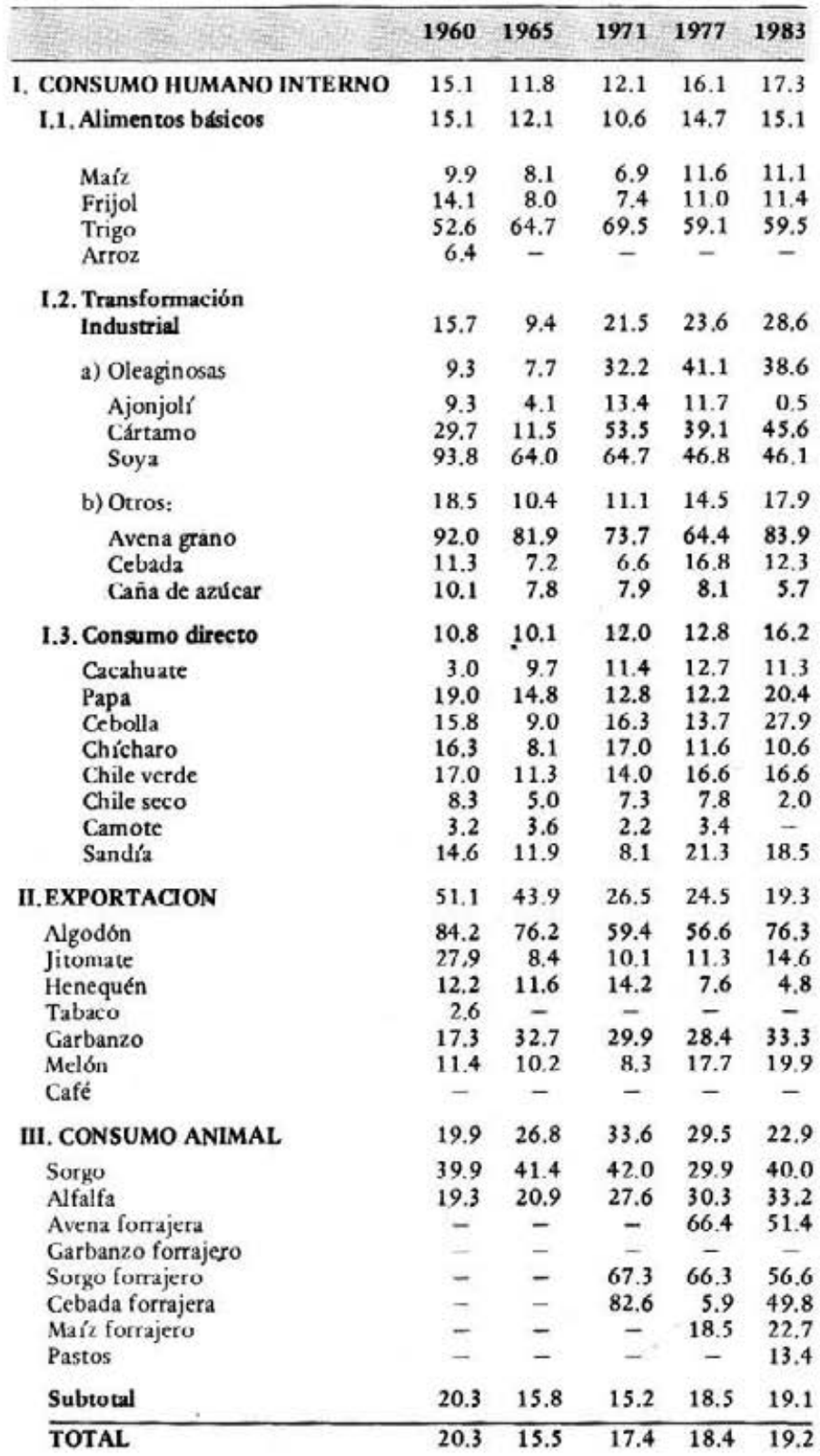


CUADRO 5. Estados fronterizos: patrón de cultivos. 1960 (hectáreas).

\begin{tabular}{|c|c|c|c|c|c|c|c|c|}
\hline & & $\begin{array}{c}\text { BAJA } \\
\text { CALIFORNIA }\end{array}$ & SONORA & CHНUAHUA & COAHUILA & $\begin{array}{l}\text { NUEVO } \\
\text { LEON }\end{array}$ & TAMAULIPAS & TOTAL \\
\hline \multicolumn{2}{|c|}{ 1. CONSUMO HUMANO INTERNO } & 71.448 & 336.876 & 504.127 & 110.728 & 136.723 & 223,908 & 1.383 .810 \\
\hline \multirow[t]{4}{*}{1.1.} & Alimentos bisicos & 46,044 & 304,691 & 419,006 & 108,064 & 131,425 & 182,662 & $1^{\prime} 191,892$ \\
\hline & $\begin{array}{l}\text { Maíz } \\
\text { Frijol }\end{array}$ & $\begin{array}{r}3.108 \\
304\end{array}$ & $\begin{array}{r}40,235 \\
8,922\end{array}$ & $\begin{array}{l}238,815 \\
124,264\end{array}$ & $\begin{array}{r}30,190 \\
2,173\end{array}$ & $\begin{array}{r}106,035 \\
6,538\end{array}$ & $\begin{array}{r}134,942 \\
45.670\end{array}$ & $\begin{array}{l}553,235 \\
187.870\end{array}$ \\
\hline & Trigo & 42,722 & 246,334 & 55,928 & 75,701 & 18,852 & 2,050 & 441,587 \\
\hline & Arrox & - & 9,200 & - & - & - & - & 9,200 \\
\hline \multirow[t]{8}{*}{1.2.} & $\begin{array}{l}\text { Transformación } \\
\text { Industrial }\end{array}$ & 23,155 & 28,056 & 75,135 & 1,948 & 2,654 & 35,475 & 166,423 \\
\hline & a) Oleaginosas & 180 & 29,970 & 1.547 & 57 & - & 638 & 30.392 \\
\hline & $\begin{array}{l}\text { Ajonjolt } \\
\text { CArtamo }\end{array}$ & $\overline{180}$ & $\begin{array}{r}18,250 \\
5,928\end{array}$ & $\begin{array}{r}25 \\
1,522\end{array}$ & -57 & $\overline{-}$ & 638 & $\begin{array}{r}18,970 \\
7,630\end{array}$ \\
\hline & Soya & - & 3,792 & - & $\overline{-}$ & $\overline{-}$ & $\overline{-}$ & 3,792 \\
\hline & b) Otros: & 22,975 & 86 & 73,588 & 1,891 & 2,654 & 34,837 & 136,031 \\
\hline & Avena grano & 502 & - & 72.953 & 82 & 200 & - & 73,737 \\
\hline & Cebada & 22,473 & 81 & 501 & 1,794 & 2,215 & 90 & 27,154 \\
\hline & Caña de azúcar & - & $s$ & 134 & is & 239 & 34,747 & 35,140 \\
\hline \multicolumn{2}{|c|}{ 1.3. Consumo directo } & 2.249 & 4,129 & 9,986 & 716 & 2,644 & 5,771 & 25,495 \\
\hline & Cacahuate & - & 248 & 2,004 & - & - & 20 & 2,272 \\
\hline & Papa & 395 & 463 & 5,320 & 51 & 2,029 & 75 & 8,333 \\
\hline & Cebolla & 88 & 229 & 237 & 156 & 437 & 1,185 & 2,332 \\
\hline & Chícharo & - & 1,247 & 148 & 2 & 4 & 149 & 1.550 \\
\hline & Chile werile & 740 & 601 & 830 & 120 & - & 3,372 & 5,663 \\
\hline & Chile seco & 960 & 169 & 750 & 4 & 18 & 73 & 1,974 \\
\hline & Caniots & 28 & 32 & 187 & 69 & 69 & 97 & 482 \\
\hline & Sandia & 38 & 1,140 & 510 & 314 & 87 & 800 & 2,889 \\
\hline \multicolumn{2}{|c|}{ II. EXPORTACION } & 116,146 & 176,765 & 96.258 & 74,400 & 18.344 & 310,536 & 792.449 \\
\hline & Alyodión & 115,419 & 165,715 & 95,300 & 73,479 & 17,656 & 289,960 & 757,529 \\
\hline & Jitomatc & 670 & 6,155 & 530 & 805 & 235 & 9,418 & 17,773 \\
\hline & Ilenequin & - & - & - & - & 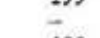 & 9,053 & 9,053 \\
\hline & Tabaco & $\cdots$ & - & - & 1 & 400 & - & 1,401 \\
\hline & Carbanzes & 30 &,+ 378 & 123 & - & 35 & 1,315 & 5,881 \\
\hline & Melión & 27 & 557 & 303 & 115 & 18 & 769 & 1,791 \\
\hline & Café & - & - & - & - & - & 21 & 21 \\
\hline \multicolumn{2}{|c|}{ II. CONSUMO ANIMAL. } & 4,767 & 9.135 & 8,504 & 3,172 & 1,440 & 36,704 & 63,722 \\
\hline & Sorgo & 165 & 6.514 & 616 & 1,014 & 1,440 & 36,704 & 46,453 \\
\hline & Alfalfa &,+ 602 & 2.621 & 7.888 & 2,158 & - & - & 17,269 \\
\hline & Avena forrajera & - & - & - & - & - & - & - \\
\hline & Garthanzo forrajero & - & - & - & - & - & - & - \\
\hline & Sorgo forrajero & - & - & - & - & - & - & - \\
\hline & Cebada forrajera & - & - & - & - & - & - & - \\
\hline & Maiz forrajero & - & - & - & - & - & - & - \\
\hline & Pastos & - & - & - & - & - & - & - \\
\hline \multicolumn{2}{|c|}{ Subtotal } & 192,361 & 522,766 & 608.889 & 188.300 & 156.507 & 571,148 & $2^{\prime} 239,981$ \\
\hline \multicolumn{2}{|c|}{ TOTAL } & 197,488 & 530,869 & 609,449 & 192,910 & 190,067 & 575,082 & 2295.862 \\
\hline
\end{tabular}


CUADRO 6. Estados fronterizos: patrón de cultivos. 1965 (hectáreas).

\begin{tabular}{|c|c|c|c|c|c|c|c|c|}
\hline \multirow{2}{*}{\multicolumn{2}{|c|}{ 1. CONSUMO HUMANO INTERNO }} & \multirow{2}{*}{$\begin{array}{c}\text { BAJA } \\
\text { CALIFORNIA } \\
76,253\end{array}$} & \multirow{2}{*}{$\begin{array}{c}\text { SONORA } \\
372.188\end{array}$} & \multirow{2}{*}{$\frac{\text { CMIUUAHUA }}{380,328}$} & COAHUILA & $\begin{array}{l}\text { NUEVO } \\
\text { LEON }\end{array}$ & TAMAULIPAS & TOTAL. \\
\hline & & & & & 129.644 & 210.669 & 256.164 & 1425.176 \\
\hline I.1. & Alimentos bísicos & 60,763 & 334,016 & 337.656 & 127,521 & 208.511 & 213,736 & 1282,176 \\
\hline & $\begin{array}{l}\text { Maiz } \\
\text { Frijol } \\
\text { Irigo }\end{array}$ & $\begin{array}{r}120 \\
60.616\end{array}$ & $\begin{array}{r}44,050 \\
3,132 \\
286834\end{array}$ & $\begin{array}{r}149,118 \\
153,085 \\
35,453\end{array}$ & $\begin{array}{r}37,760 \\
3,646 \\
86,115\end{array}$ & $\begin{array}{r}168,549 \\
9,153 \\
30,809\end{array}$ & $\begin{array}{r}211,567 \\
1,470 \\
699\end{array}$ & $\begin{array}{l}611,164 \\
170,486 \\
500,526\end{array}$ \\
\hline & $\begin{array}{l}\text { Trigo } \\
\text { Arroz }\end{array}$ & 60,616 & 286,834 & 35,453 & 86,115 & $\begin{array}{c}30,809 \\
-\end{array}$ & 699 & 500,526 \\
\hline \multirow[t]{7}{*}{1.2.} & $\begin{array}{l}\text { Transformación } \\
\text { Industrial }\end{array}$ & 13,021 & 35,532 & 30,409 & 1,428 & 421 & 37,863 & 118.674 \\
\hline & a) Oleaginosas & 1,158 & 32,185 & 343 & 1,025 & - & 830 & 35,541 \\
\hline & $\begin{array}{l}\text { Ajonjoli } \\
\text { Círtamo } \\
\text { Soya }\end{array}$ & $\overline{1,158}$ & $\begin{array}{r}10,279 \\
4,337 \\
-\end{array}$ & $\begin{array}{r}12 \\
331 \\
-\end{array}$ & $\begin{array}{l}50 \\
975 \\
-\end{array}$ & $\bar{z}$ & $\begin{array}{l}830 \\
= \\
-\end{array}$ & $\begin{array}{r}11,171 \\
6,801 \\
17,569\end{array}$ \\
\hline & b) Otros: & 11.863 & 3,347 & 30,066 & 403 & 421 & 37,033 & 83,133 \\
\hline & Avena grano & - & - & 30,000 & - & $=$ & - & 30.000 \\
\hline & Cebada & 11,863 & 3,347 & - & 403 & 421 & 391 & 16,425 \\
\hline & Caña de azúcar & - & - & 66 & - & - & 36,642 & 36,708 \\
\hline \multicolumn{2}{|c|}{ 1,3. Consamo directo } & 2,496 & 2,570 & 12,263 & 695 & 1.737 & 4,565 & 24,326 \\
\hline & Cacahuate & - & 16 & 6.243 & - & - & - & 6.259 \\
\hline & Papa & 683 & 165 & 3.625 & 45 & 1.261 & 25 & 5.804 \\
\hline & Cebolla & 6 & 93 & 456 & 66 & 144 & -742 & 1,467 \\
\hline & Chichero & - & 891 & 73 & - & $2-$ & - & 966 \\
\hline & Chile erde & 765 & 168 & 987 & - & - & 2,823 & 4,743 \\
\hline & Chile seco & 999 & 101 & 75 & - & - & - & 1.175 \\
\hline & Camote & - & 53 & 372 & 28 & 55 & ' & 508 \\
\hline & Sandia & 43 & 1,123 & 432 & 556 & 275 & 975 & 3.404 \\
\hline \multicolumn{2}{|c|}{ II. EXPORTACION } & 107,910 & 173,513 & 64,058 & 62,296 & 1,530 & 242,447 & 651.754 \\
\hline & Nggodón & 107.855 & 170.821 & 63.296 & 61.362 & 1,084 & 215,117 & 619.535 \\
\hline & Jitomate & ss & 1,314 & 392 & 701 & 360 & 941 & 3.763 \\
\hline & Henequen & - & - & - & - & - & 23,456 & 23,456 \\
\hline & Talaro & - & - & - & - & $\begin{array}{l}- \\
\text { - }\end{array}$ & - & - \\
\hline & Garhanzo & - & 1,133 & 105 & - & 61 & 1,768 & 3,067 \\
\hline & Melón & - & 245 & 265 & 233 & 25 & 1,165 & 1.933 \\
\hline & Cafe & - & - & - & - & - & - & - \\
\hline \multicolumn{2}{|c|}{ III. CONSUMO ANIMAL } & 5,496 & 13,249 & 15,228 & 7,065 & 13,086 & 97,851 & 151.975 \\
\hline & Sorgo & - & 10,867 & 4,037 & 3,908 & 13,086 & 97,851 & 129,749 \\
\hline & Alfalfa & 5,496 & 2,382 & 11,191 & 3,157 & - & - & 22,260 \\
\hline & Avena forraje & - & - & - & - & - & - & - \\
\hline & Garbanzo forrajero & $\underline{2}$ & - & - & - & - & - & - \\
\hline & Sorgo forrajero & - & - & - & - & - & - & - \\
\hline & Cebada forrajera & - & - & - & - & - & - & - \\
\hline & Maí, forrajero & - & - & - & - & - & - & - \\
\hline & Pastos & - & - & - & - & - & - & - \\
\hline \multicolumn{2}{|c|}{ Subtotal } & 189.659 & 558.880 & 459.614 & 199.005 & 225.285 & 596,462 & 2'228.905 \\
\hline \multicolumn{2}{|c|}{ TOTAL } & 189,700 & 567,000 & 460,600 & 207.555 & 226,300 & 623,400 & 2274.555 \\
\hline
\end{tabular}


CUADRO 7. Estados fronterizos: patrón de cultivos. 1971 (hectáreas).

\begin{tabular}{|c|c|c|c|c|c|c|c|c|}
\hline \multirow{2}{*}{\multicolumn{2}{|c|}{ 1. CONSUMO HUMANO INTERNO }} & \multirow{2}{*}{$\begin{array}{c}\text { BAJA } \\
\text { CALIFORNIA }\end{array}$} & \multirow{2}{*}{$\frac{\text { SONORA }}{431,938}$} & \multirow{2}{*}{$\begin{array}{c}\text { CHIHUAHUA } \\
496,955\end{array}$} & \multirow{2}{*}{$\begin{array}{c}\text { СОАНUILA } \\
62,518\end{array}$} & \multirow{2}{*}{$\begin{array}{r}\begin{array}{r}\text { NUEVO } \\
\text { LEON }\end{array} \\
76,422 \\
\end{array}$} & \multirow{2}{*}{$\begin{array}{c}\text { TAMAULIPAS } \\
370,842\end{array}$} & \multirow{2}{*}{$\begin{array}{r}\text { TOTAL } \\
1483,049\end{array}$} \\
\hline & & & & & & & & \\
\hline \multirow{4}{*}{ I.1, } & Alimentes bisicos & 58,262 & 240,147 & 386,846 & 52,834 & 73.693 & 289,754 & 1'101,536 \\
\hline & $\begin{array}{l}\text { Maiz } \\
\text { Frijol }\end{array}$ & 3.896 & $\begin{array}{r}11,869 \\
1,281\end{array}$ & $\begin{array}{l}169,499 \\
127,842\end{array}$ & $\begin{array}{r}16.719 \\
5,056\end{array}$ & $\begin{array}{r}52.000 \\
2,693\end{array}$ & $\begin{array}{r}277.575 \\
6.179\end{array}$ & $\begin{array}{l}531.558 \\
143,051\end{array}$ \\
\hline & Trigo & 54,366 & 226,997 & 89,505 & 31,509 & 19,000 & 6,000 & $\begin{array}{l}143,051 \\
426,927\end{array}$ \\
\hline & Arroz & - & - & - & - & - & - & - \\
\hline & $\begin{array}{l}\text { Transformación } \\
\text { Industrial }\end{array}$ & 33.365 & 186.558 & 46.638 & 8.770 & 993 & 77.122 & 353,446 \\
\hline & a) Oleaginosas & 21,807 & 185,512 & 6,415 & 7,370 & 5 & 39,400 & 260.509 \\
\hline & Ajonjoli & $\therefore$ & 30,702 & 15 & - & 5 & 7,000 & 37,722 \\
\hline & Cártamo & 21,807 & $\begin{array}{l}94,441 \\
60,369\end{array}$ & $\begin{array}{l}1,200 \\
5,200\end{array}$ & 7,370 & $\overline{-}$ & $\begin{array}{l}15,000 \\
17,400\end{array}$ & $\begin{array}{r}139,818 \\
82,969\end{array}$ \\
\hline & Soya & - & 60,369 & & & & & 82,969 \\
\hline & b) Otros: & 11.558 & 1,046 & 40.223 & 1,400 & 988 & 37.722 & 92,937 \\
\hline & Avena grano & 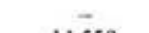 & - & 40,223 & 200 & - & - & 40.423 \\
\hline & Cebada & 11,558 & 986 & $z$ & 1,200 & 888 & $-\overline{7}$ & 14,632 \\
\hline & Caña de azúcar & - & 60 & & - & 100 & 37,722 & 37,882 \\
\hline \multirow[t]{9}{*}{1.3.} & Consumo directo & 2.747 & 5.233 & 13.471 & 914 & 1,736 & 3,966 & 28,067 \\
\hline & Cacahuate & - & 203 & 6,531 & - & - & - & 6.734 \\
\hline & Papa & 1.150 & $\begin{array}{r}588 \\
28\end{array}$ & 2,566 & 163 & 1.500 & - & 5,967 \\
\hline & Cebolla & - & $\begin{array}{r}28 \\
2.185\end{array}$ & 1,200 & 65 & 220 & 2.000 & 3,513 \\
\hline & Chicharo & 1.475 & $\begin{array}{l}2.185 \\
1.521\end{array}$ & 82 & - & - & 40 & 2,307 \\
\hline & Chile verde & 1.475 & 1.521 & 1,300 & +33 & - & 1,500 & 6,229 \\
\hline & Chile seco & - & 62 & 1,300 & - & - & - & 1,362 \\
\hline & Camote & - & 17 & 242 & - & - & - & 259 \\
\hline & Sandía & 122 & 629 & 250 & 253 & 16 & 426 & 1.696 \\
\hline \multicolumn{2}{|c|}{ I.EXPORTACION } & 52.892 & 142,031 & 31,765 & 52.812 & 244 & 30,940 & $310,6 \times 4$ \\
\hline & Algodón & 51,499 & 136,569 & 30,405 & 52.253 & 18 & 1,288 & 272.032 \\
\hline & Jitomate & 1,350 & 1,068 & 1,100 & 317 & 200 & 2,189 & 6,224 \\
\hline & Henequén & - & - & - & - & - & 25,963 & 25,963 \\
\hline & Tabaco & - & $=$ & - & - & 20 & - & 20 \\
\hline & Garbanzo & - & 4,066 & 110 & - & - & 800 & 4.976 \\
\hline & Melón & 43 & 328 & 150 & 242 & 6 & 700 & 1,469 \\
\hline & Cafe & - & - & - & - & - & - & - \\
\hline \multirow{10}{*}{ II. CON } & ONSUMO ANIMAL & 22,883 & 32,510 & 36.500 & 21.110 & 16.216 & 316,888 & 446.107 \\
\hline & Sorgo & 6,531 & 22,437 & 22,000 & 12,000 & 15,616 & 315,016 & 392,600 \\
\hline & Alfalfa & 15,677 & 10,073 & 14,500 & 5,042 & - & - & 45.292 \\
\hline & $\begin{array}{l}\text { Avena forrajera } \\
\text { Garbanzo forrajero }\end{array}$ & - & $\overline{-}$ & $\overline{-}$ & 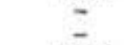 & $\bar{z}$ & $\overline{-}$ & $\bar{z}$ \\
\hline & Sorgo forrajero & 675 & - & - & 2.718 & 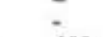 & 1.872 & 5,265 \\
\hline & Cebada forrajera & - & - & - & 1.350 & 600 & - & 1,950 \\
\hline & Maít forrajero & $\bar{z}$ & $\overline{-}$ & $=$ & $\overline{-}$ & - & - & \\
\hline & Pastos & - & - & - & - & - & - & - \\
\hline & Subtotal & 170.149 & 606.459 & 515,170 & 136,440 & 92,882 & 718,670 & 2239,770 \\
\hline & TOTAL. & 227,400 & 772,800 & 746.700 & 203.300 & 151,100 & $734,9 \times 5$ & $2^{4} \times 36,285$ \\
\hline
\end{tabular}


CUADRO 8. Estados fronterizos: patrón de cultivos. 1977 (hectáreas).

\begin{tabular}{|c|c|c|c|c|c|c|c|c|}
\hline & & $\begin{array}{c}\text { BAJA } \\
\text { CALIFORNIA }\end{array}$ & SONORA & CHHUAHUA & COAHUILA & $\begin{array}{l}\text { NUEVO } \\
\text { LEON }\end{array}$ & TAMAULIPAS & TOTAL \\
\hline \multicolumn{2}{|c|}{ 1. CONSUMO HUMANO INTERNO } & 111,061 & 396,067 & 518,247 & 93.042 & 113.394 & 716,147 & $1 ' 954,958$ \\
\hline \multirow[t]{4}{*}{1.1.} & Alimentos bisicos & 73,777 & 269,315 & 443,237 & 78,588 & 110,955 & 493.486 & 1469,358 \\
\hline & Maíz & $\begin{array}{r}1,899 \\
278\end{array}$ & $\begin{array}{r}19,565 \\
2,250\end{array}$ & $\begin{array}{l}231,222 \\
136,713\end{array}$ & $\begin{array}{r}42,483 \\
8613\end{array}$ & 89,585 & $\begin{array}{r}482,840 \\
10441\end{array}$ & 867,594 \\
\hline & $\begin{array}{l}\text { Fnjol } \\
\text { Ingo }\end{array}$ & 71.600 & 247,500 & 65.302 & 27.492 & 9,508 & 205 & $\begin{array}{l}180,157 \\
421,607\end{array}$ \\
\hline & Arroz & - & - & - & - & & & \\
\hline \multirow[t]{9}{*}{1.2 . } & Transformación & & & & & & & \\
\hline & Industrial & 39.667 & 119,413 & 58,845 & 13,330 & 559 & 220,030 & 451,844 \\
\hline & a) Oleaginosas & 8.115 & 118,072 & 16,388 & 5,073 & 60 & 181,477 & 329,185 \\
\hline & $\begin{array}{l}\text { Ajonjoli' } \\
\text { Cartamo }\end{array}$ & $\begin{array}{r}415 \\
7,700\end{array}$ & $\begin{array}{l}22,611 \\
72,093\end{array}$ & $-\overline{881}$ & 5,073 & 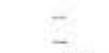 & $\begin{array}{r}1,038 \\
72,300\end{array}$ & $\begin{array}{r}24,064 \\
158,047\end{array}$ \\
\hline & Soya & - & 23,368 & 15,507 & - & 60 & 108,139 & 147,074 \\
\hline & b) Otros: & 31.552 & 1.341 & 42.457 & 8,257 & 499 & 38,553 & 122,659 \\
\hline & Avena grano & 452 & 87 & 41,580 & 1.150 & - & - & 43.269 \\
\hline & Crlada & 31,100 & 1,254 & 877 & 7,107 & 499 & 900 & 41.737 \\
\hline & Caña de azúcar & - & - & - & - & - & 37,653 & 37,653 \\
\hline \multirow[t]{9}{*}{1.3 . } & Consumo directo & 4,617 & 7.339 & 16,165 & 1,124 & 1,880 & 2,631 & 33,756 \\
\hline & Cacahuate & - & 563 & 5.500 & - & - & - & 6,063 \\
\hline & Papa & 747 & 432 & 3.905 & 70 & 1,460 & 1 & 6,615 \\
\hline & Cebolla & 116 & 229 & 1,572 & 118 & 100 & 751 & 2,886 \\
\hline & Chícharo & - & 1,982 & - & - & - & 406 & 2,388 \\
\hline & Chile verde & - & 2,403 & 4,638 & 312 & 240 & 712 & 8,305 \\
\hline & Chile seco & 2.398 & 369 & - & - & - & - & 2,767 \\
\hline & Camote & - & $\overline{7}$ & 250 & - & - & - & 250 \\
\hline & Sandía & 1,356 & 1,361 & 300 & 624 & 80 & 761 & 4,482 \\
\hline \multicolumn{2}{|c|}{ II.EXPORTACION } & 56,821 & 128,833 & 34,118 & 50,211 & 90 & 26,267 & 296,340 \\
\hline & Algodón & 52.588 & 106.727 & 32,828 & 49,415 & - & 10,395 & 251,953 \\
\hline & Jitomate & 2,427 & 1,119 & 990 & 544 & 60 & 1,847 & 6,977 \\
\hline & $\begin{array}{l}\text { Henequén } \\
\text { Tabaco }\end{array}$ & $\overline{-}$ & 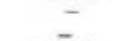 & - & - & - & 13,420 & 13,420 \\
\hline & $\begin{array}{l}\text { Tabaco } \\
\text { Garbanzo }\end{array}$ & $\overline{-}$ & $20, \overline{812}$ & $\bar{z}$ & $\overline{-}$ & _- & - & 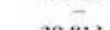 \\
\hline & $\begin{array}{l}\text { Garbanzo } \\
\text { Fresa }\end{array}$ & - & 4 & $=$. & $\overline{-}$ & $\overline{-}$ & 1 & 20,813 \\
\hline & $\begin{array}{l}\text { Fresá } \\
\text { Melón }\end{array}$ & 1.816 & 171 & 300 & 252 & $\overline{-}_{30}$ & $\overline{6}$ & $33^{4}$ \\
\hline & Café & - & - & - & - & - & ${ }_{-}^{604}$ & $\stackrel{5,175}{-}$ \\
\hline \multirow[t]{10}{*}{ III. $C$} & ONSUMO ANIMAL. & 19,682 & 27,590 & 106,670 & 46,978 & $\$ 1.701$ & 325.715 & 578.336 \\
\hline & Sorgo & 1.512 & 13,028 & 22,747 & 15,162 & 45,856 & 323,938 & 422,243 \\
\hline & Alfalfa & 15,934 & 13,562 & 22,000 & 9,261 & 2,836 & 20 & 61,613 \\
\hline & Avena forrajera & & - & 58.827 & 8,056 & 560 & 80 & 67,523 \\
\hline & $\begin{array}{l}\text { Garbanzo forrajero } \\
\text { Sorgo forraicro }\end{array}$ & $2, \overline{236}$ & $9, \overline{70}$ & 2069 & 7,570 & $\overline{1.819}$ & $\overline{4}$ & - \\
\hline & $\begin{array}{l}\text { Sorgo forrajero } \\
\text { Cebada forrajera }\end{array}$ & $\stackrel{2,236}{-}$ & $\begin{array}{r}9,700 \\
30\end{array}$ & $\begin{array}{c}2,069^{\prime} \\
177\end{array}$ & $\begin{array}{l}7,570 \\
3,927\end{array}$ & $\begin{array}{r}1,819 \\
635\end{array}$ & 1.677 & $\begin{array}{r}16,336 \\
4,769\end{array}$ \\
\hline & $\begin{array}{l}\text { Cebada forrajcra } \\
\text { Maíz forrajero }\end{array}$ & - & - & 850 & 3,002 & 635 & $\bar{z}$ & $\begin{array}{l}4,769 \\
3,852\end{array}$ \\
\hline & Pastos & - & - & - & 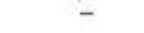 & - & - & - \\
\hline & Subtotal & 194,564 & $\$ \$ 2,490$ & 659,035 & 190.231 & 165,185 & $1^{\prime} 068,129$ & $2^{\prime} 829,634$ \\
\hline & TOTAL & 222,574 & 585,490 & 689,963 & 226,190 & 238.752 & $1 ' 113.729$ & 37076,698 \\
\hline
\end{tabular}


CUADRO 9. Estados fronterizos: patrón de cultivos. 1979 (hectáreas).

\begin{tabular}{|c|c|c|c|c|c|c|c|}
\hline & $\begin{array}{c}\text { BAJA } \\
\text { CALIFORNIA }\end{array}$ & SONORA & СНIHUAHUA & COAHUILA & $\begin{array}{l}\text { NUEVO } \\
\text { LEON }\end{array}$ & TAMAULIPAS & TOTAL \\
\hline L, CONSUMO HUMANO INTERNO & 133,843 & 536,537 & 447,834 & 60,553 & 79,610 & 422,488 & 1680,865 \\
\hline 1. 1. Alimentos bdsicos & 66,824 & 198,921 & 389.793 & 46,830 & 75,615 & 245.116 & 1023,099 \\
\hline Maiz & 9.419 & $\begin{array}{r}26,576 \\
5,949\end{array}$ & $\begin{array}{l}217,460 \\
120,393\end{array}$ & $\begin{array}{r}27,002 \\
4,414\end{array}$ & 51,721 & 221,614 & 553.792 \\
\hline $\begin{array}{l}\text { Frijol } \\
\text { Trigo }\end{array}$ & $\begin{array}{r}1,195 \\
56,210\end{array}$ & $\begin{array}{r}5,949 \\
166,396\end{array}$ & $\begin{array}{r}120,393 \\
51,940\end{array}$ & $\begin{array}{r}4,414 \\
15,414\end{array}$ & $\begin{array}{r}3,771 \\
20,123\end{array}$ & $\begin{array}{r}22,059 \\
851\end{array}$ & $\begin{array}{l}157.781 \\
310.934\end{array}$ \\
\hline $\begin{array}{l}\text { Trigo } \\
\text { Arroz }\end{array}$ & $\begin{array}{c}56,210 \\
-\end{array}$ & $\begin{array}{c}166,390 \\
-\end{array}$ & $\begin{array}{c}51,940 \\
-\end{array}$ & 15,414 & $\begin{array}{l}20,123 \\
-\end{array}$ & 592 & $\begin{array}{r}310,934 \\
592\end{array}$ \\
\hline $\begin{array}{l}\text { 1. 2. Transformación } \\
\text { Industrial }\end{array}$ & 61.174 & 328,675 & 40,560 & 12.130 & 2.688 & 172.973 & 618.200 \\
\hline a) Oleaginosas & 19,968 & 327,754 & 23,811 & 7,832 & 1.269 & 142,419 & 522,783 \\
\hline $\begin{array}{l}\text { Ajonjolr } \\
\text { Círtamo }\end{array}$ & $\begin{array}{r}6,286 \\
.13,682\end{array}$ & $\begin{array}{r}93,130 \\
127.158\end{array}$ & $\overline{1.182}$ & $\overline{7,813}$ & $\overline{607}$ & $\begin{array}{r}434 \\
82,480\end{array}$ & $\begin{array}{r}99,870 \\
232,922\end{array}$ \\
\hline Soya & - & 107,446 & 22,629 & 19 & 662 & 59.235 & 189.991 \\
\hline b) Otros: & 41,206 & 921 & 16,749 & 4,298 & 1,419 & 20,824 & 95,417 \\
\hline $\begin{array}{l}\text { Avena grano } \\
\text { Cebada }\end{array}$ & $\begin{array}{r}1,207 \\
39.999\end{array}$ & $\overline{879}$ & $\begin{array}{r}15,469 \\
1,280\end{array}$ & $\begin{array}{l}1,409 \\
2,889\end{array}$ & $\begin{array}{r}73 \\
1,295\end{array}$ & $\overline{750}$ & $\begin{array}{r}18,158 \\
47,092\end{array}$ \\
\hline Caña de azùcar & - & 42 & 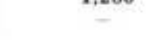 & $+2,00$ & 51 & 30.074 & 30.167 \\
\hline 1. 3. Consumo Directo & 5,845 & 8,941 & 17,481 & 1,593 & 1,307 & 4,399 & 39,566 \\
\hline Cacabuatc & - & +72 & 4,798 & - & 109 & 51 & 5,430 \\
\hline Papa & 2,115 & 2,791 & 6.239 & 595 & 1,083 & 31 & 12,854 \\
\hline Cebolla & 1.923 & 643 & 2.293 & 12 & - & 1,616 & 6,487 \\
\hline Chicharo & 85 & 2,060 & - & - & & 30 & 2.175 \\
\hline Chile verde & 781 & 1,189 & 3,511 & 569 & 18 & 1.694 & 7.762 \\
\hline Chile seco & - & 170 & 264 & 15 & - & - & 449 \\
\hline Camote & - & 1 & 168 & 22 & - & - & 191 \\
\hline Sandía & 941 & 1,615 & 208 & 380 & 97 & 977 & 4,218 \\
\hline I.EXPORTACION & 62,485 & 156,540 & 39,615 & 45,840 & 212 & 22,730 & 327,422 \\
\hline Algodón & 56,842 & 98,616 & 38,987 & 44,836 & - & 2,600 & 241,881 \\
\hline $\begin{array}{l}\text { Jitomate } \\
\text { Henequedn }\end{array}$ & 3,952 & 902 & 311 & 340 & 92 & 3,352 & 10,949 \\
\hline $\begin{array}{l}\text { Henequein } \\
\text { Tataco }\end{array}$ & - & - & - & - & - & 14,000 & 14,000 \\
\hline $\begin{array}{l}\text { Tabaco } \\
\text { Garbanzo }\end{array}$ & - & - & - & - & - & - & - \\
\hline $\begin{array}{l}\text { Garbanzo } \\
\text { Melón }\end{array}$ & 13 & 56,814 & so & - & 46 & 9 & 56.932 \\
\hline $\begin{array}{l}\text { Melón } \\
\text { Café }\end{array}$ & 1,678 & 208 & 267 & 664 & 79 & 769 & 3.660 \\
\hline & - & - & - & - & - & - & - \\
\hline II. CONSUMO ANIMAL & 42,393 & 34.138 & 170.995 & 43,314 & 31,070 & 506,731 & 848,641 \\
\hline Sorgo & 5,703 & 12,809 & 17,928 & 8.421 & 38.009 & 499.756 & 582.626 \\
\hline Alfalfa & 17,912 & 15,837 & 26,358 & 11,669 & 3,986 & 62 & 75.824 \\
\hline Avena forrajera & 1,386 & 143 & 118,158 & 9,262 & 1,581 & 78 & 130,808 \\
\hline Garbanzo forrajero & - & - & - & - & - & - & - \\
\hline Sorgo forrajeró & 3,134 & 829 & 1.395 & 6,583 & 1,474 & 1,383 & 14,798 \\
\hline Cebuda forrajera & 3,040 & 1.752 & 22 & 932 & 1,266 & - & 7,012 \\
\hline Maí forrajero & - & 40 & 5,966 & 2.023 & 1.709 & - & 9.738 \\
\hline Pastos & 11,018 & 2,728 & 1,168 & 4,424 & 3,045 & 5.452 & 27.835 \\
\hline Subtotal & 238,721 & 727.215 & 658,444 & 149,709 & 130,892 & 951,949 & $2 * 856,930$ \\
\hline Total & 259,222 & 758.051 & 690.912 & 172,832 & 196,725 & 996,449 & $3^{\prime} 074,191$ \\
\hline
\end{tabular}




\section{CUADRO 10. Estados fronterizos: patrón de cultivos. 1983 (hectáreas).}

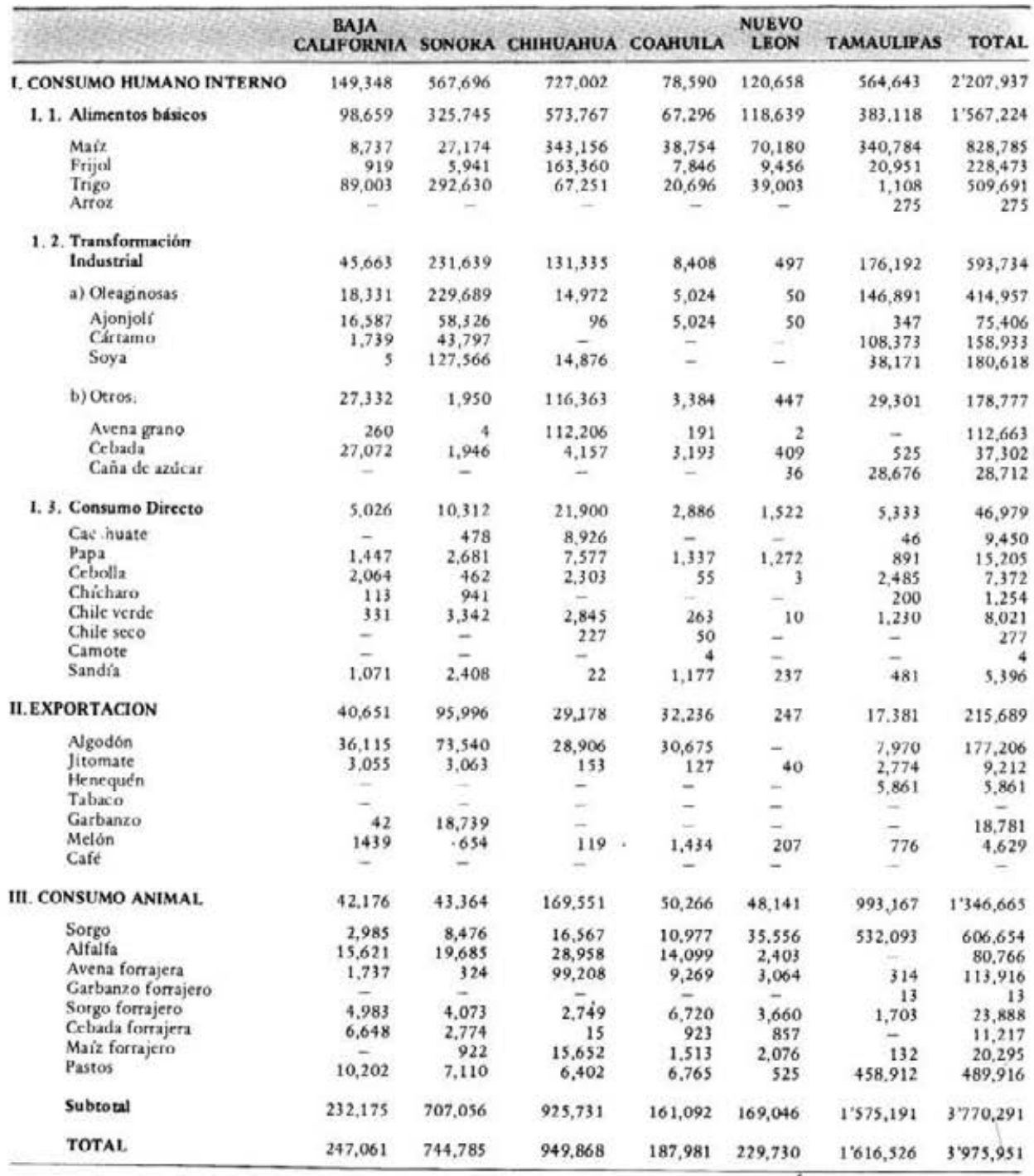


CUADRO 11. Estados fronterizos: patrón de cultivos (porcentajes).

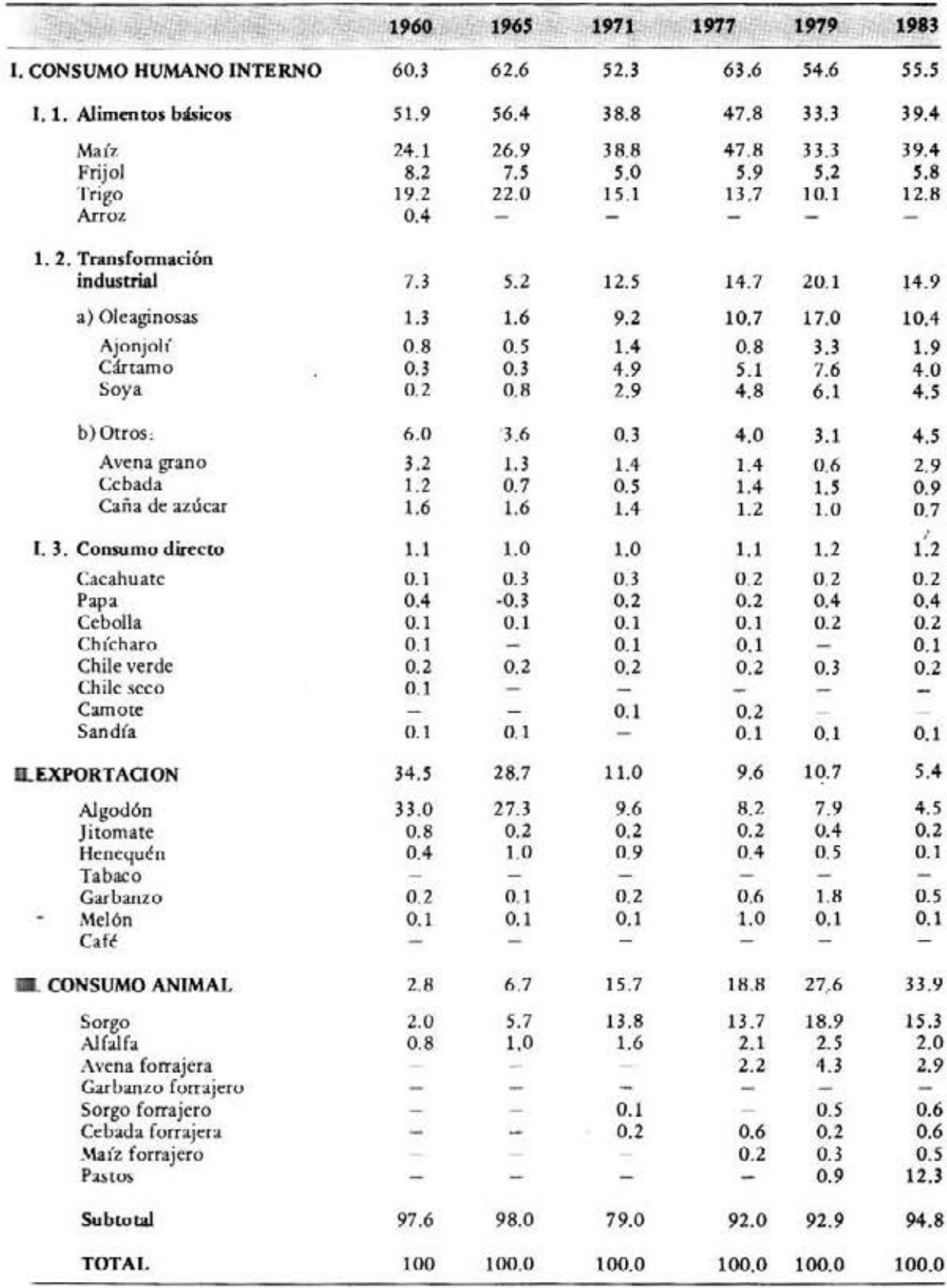


CUADRO 12. Baja California: patrón de cultivos. (porcentajes).

\begin{tabular}{|c|c|c|c|c|c|c|}
\hline & 1960 & 1965 & 1971 & 1977 & 1979 & 1983 \\
\hline I. CONSUMO HUMANO INTERNO & 36.2 & 40.2 & 41.5 & 53.1 & 51.6 & 60.5 \\
\hline I. 1. Alimentos bisicos & 23.3 & 32.0 & 25.6 & 33.2 & 25.8 & 40.0 \\
\hline Marz & 1.5 & - & 1.7 & 0.9 & 3.6 & 3.6 \\
\hline Frijol & 0.2 & - & - & 0.1 & 0.5 & 0.4 \\
\hline Trigo & 21.6 & 32.0 & 23.9 & 32.2 & 21.7 & 36.0 \\
\hline Arroz & - & - & - & - & - & - \\
\hline \multicolumn{7}{|l|}{ 1. 2. Transformación } \\
\hline industrial & 11.7 & 6.9 & 14.7 & 17.8 & 23.6 & 18.5 \\
\hline a) Oleaginosas & 0.1 & 0.6 & 9.6 & 3.6 & 7.7 & 7.4 \\
\hline Ajonjolí & - & - & - & 0.2 & 2.4 & 6.7 \\
\hline Cártamo & 0.1 & 0.6 & 9.6 & 3.4 & 5.3 & 0,7 \\
\hline Soya & - & - & - & - & - & - \\
\hline b) Otros: & 11.6 & 6.3 & 5.1 & 14.2 & 15.9 & 11.1 \\
\hline Avena grano & 0.2 & - & - & 0.2 & 0.5 & 0.1 \\
\hline Cebada & 11.4 & 6.3 & 5.1 & 14.0 & 15.4 & 11.0 \\
\hline Caña de azúcar & - & - & - & - & - & - \\
\hline I. 3. Consumo directo & 1.2 & 1.3 & 1.2 & 2.1 & 2.2 & 2.0 \\
\hline Cacahuate & - & - & - & - & - & - \\
\hline Papa & 0.2 & 0.4 & 0.5 & 0.3 & 0.8 & 0.6 \\
\hline Cebolla & 0.1 & - & - & - & - & 0.1 \\
\hline Chícharo & - & - & - & - & - & 0.1 \\
\hline Chile verde & 0.4 & 0.4 & 0.6 & - & - & 0.1 \\
\hline Chile seco & 0.5 & 0.5 & - & 1.1 & 0.3 & - \\
\hline Camote & - & - & - & - & - & - \\
\hline Sandía & - & - & 0.1 & 0.6 & 0.4 & 0.4 \\
\hline II.EXPORTACION & 58.8 & 56.9 & 23.3 & 25.5 & 24.1 & 16.5 \\
\hline Algodón & 58.8 & 56.9 & 22.7 & 23.6 & 21.9 & 14.6 \\
\hline Jitomate & 0.3 & - & . $\quad 0.6$ & 1.1 & 1.5 & 1.3 \\
\hline Henequén & - & - & - & - & - & - \\
\hline Tabaco & - & - & - & - & - & - \\
\hline Garbanzo & - & - & - & - & - & - \\
\hline Melón & - & - & - & 0.8 & 0.7 & 0.6 \\
\hline Café & - & - & - & - & - & - \\
\hline III. CONSUMO ANIMAL & 2.4 & 2.9 & 10.0 & 8.8 & 16.4 & 17.0 \\
\hline Sorgo & 0.1 & - & 2.9 & 0.7 & 2.2 & 1.2 \\
\hline Alfalfa & 2.3 & 2.9 & 6.9 & 7.1 & 6.9 & 6.3 \\
\hline Avena forrajera & - & - & - & - & 0.6 & 0.7 \\
\hline Garbanzo forrajero & - & - & - & - & - & - \\
\hline Sorgo forrajero & - & - & - & - & 1.2 & 2.0 \\
\hline Cebada forrajera & - & - & 0.2 & 1.0 & 1.2 & 2.7 \\
\hline Maíz forrajero & - & - & - & - & - & - \\
\hline Pastos & - & - & - & - & 4.3 & 4.1 \\
\hline Subtotal & 97.4 & 100.0 & 74.8 & 87.4 & 92.1 & 94.0 \\
\hline TOTAL & 100 & 100.0 & 100.0 & 100.0 & 100.0 & 100,0 \\
\hline
\end{tabular}


CUADRO 13. Sonora: patrón de cultivos.

\begin{tabular}{|c|c|c|c|c|c|c|}
\hline & 1960 & 1965 & 1971 & 1977 & 1979 & 1983 \\
\hline I. CONSUMO HUMANO INTERNO & 62.5 & 65.6 & 55.9 & 67.7 & 70.8 & 76.2 \\
\hline 1. 1. Alimentos básicos & 57.4 & 59.0 & 31.1 & 46.0 & 26.2 & 43.7 \\
\hline Maíz & 7.6 & 7.8 & 1.5 & 3.3 & 3.5 & 3.7 \\
\hline Frijol & 1.7 & 0.6 & 0.2 & 0.4 & 0.8 & 0.8 \\
\hline Trigo & 46.4 & 50.6 & 29.4 & 42.3 & 21.9 & 39.2 \\
\hline Arroz & 1.7 & - & - & - & - & - \\
\hline $\begin{array}{l}\text { 1. 2. Transformación } \\
\text { industrial }\end{array}$ & 5.3 & 6.2 & 24.1 & 20.4 & 43.4 & 31.1 \\
\hline a) Oleaginosas & 5.3 & 5.6 & 24.0 & 20.2 & 43.3 & 30.8 \\
\hline $\begin{array}{l}\text { Ajonjolí } \\
\text { Cártamo }\end{array}$ & $\begin{array}{l}3.5 \\
1.10 .8\end{array}$ & 1.8 & $\begin{array}{r}4.0 \\
12.2\end{array}$ & $\begin{array}{r}3.9 \\
12.3\end{array}$ & $\begin{array}{l}12.3 \\
16.8\end{array}$ & $\begin{array}{l}7.8 \\
5.9\end{array}$ \\
\hline Soya & 0.7 & 3.0 & 7.8 & 4.0 & 14.2 & 17,1 \\
\hline b) Otros: & - & 0.6 & 0.1 & 0.2 & 0.1 & 0.3 \\
\hline Avena grano & - & $\overline{0}$ & - & - & - & - \\
\hline Cebada & - & 0.6 & 0.1 & 0.2 & 0.1 & 0.3 \\
\hline Caña de azúcar & - & - & - & - & - & - \\
\hline I. 3. Consumo directo & 0.8 & 0.4 & 0.7 & 1.3 & 1.2 & 1.4 \\
\hline Cacahuate & 0.1 & - & - & 0.1 & 0.1 & 0,1 \\
\hline Papa & 0.1 & - & 0,1 & 0.1 & 0.3 & 0,4 \\
\hline Cebolla & 0.1 & - & - & $=$ & 0.1 & 0.1 \\
\hline Chícharo & 0.2 & 0.2 & 0.3 & 0.3 & 0,3 & 0.1 \\
\hline Chile verde & 0.1 & - & 0.2 & 0.4 & 0.2 & 0.4 \\
\hline Chile seco & - & - & - & - & - & - \\
\hline Camote & $\overrightarrow{0}$ & $\overrightarrow{0}$ & $\overline{0}$ & - & - & - \\
\hline Sandía & 0.2 & 0.2 & 0.1 & 0.4 & 0,2 & 0.3 \\
\hline II.EXPORTACION & 33.3 & 30.6 & 18.4 & 22.0 & 20.6 & 12.9 \\
\hline Algodón & 31.2 & 30.2 & 17.7 & 18.2 & 13.0 & 9.9 \\
\hline Jitomate & 1.2 & 0.2 & 0.1 & 0.2 & 0.1 & 0.4 \\
\hline Henequén & - & - & - & - & - & - \\
\hline Tabaco & - & - & - & $\overline{2}$ & $\bar{z}$ & $\bar{s}$ \\
\hline Garbanzo & 0.8 & 0.2 & 0.5 & 3.6 & 7.5 & 2,5 \\
\hline Melón & 0.1 & - & - & - & - & 0.1 \\
\hline Café & - & - & - & - & - & - \\
\hline III. CONSUMO ANIMAL & 1.7 & 2.3 & 4.2 & 4.7 & 4.5 & 5.8 \\
\hline Sorgo & 1.2 & 1.9 & 2.9 & 2.2 & 1.7 & 1.1 \\
\hline Alfalfa & 0.5 & 0.4 & 1.3 & 2.3 & 2,1 & 2.6 \\
\hline Avena forrajera & - & - & - & - & - & 0.1 \\
\hline Garbanzo forrajero & - & - & - & - & - & - \\
\hline Sorgo forrajero & - & - & - & $\overline{0}$ & 0.1 & 0.5 \\
\hline Cebada forrajera & - & - & - & 0.2 & 0.2 & 0.4 \\
\hline Maíz forrajero & - & - & - & - & 0.4 & 0.1 \\
\hline Pastos & - & - & - & - & - & 1.0 \\
\hline Subtotal & 98.5 & 98.5 & 78.5 & 94.4 & 95.9 & 94.9 \\
\hline TOTAL & 100.0 & 100.0 & 100.0 & 100.0 & 100.0 & 100,0 \\
\hline
\end{tabular}


CUADRO 14. Chihuahua: patrón de cultivos (porcentajes).

\begin{tabular}{|c|c|c|c|c|c|c|c|}
\hline & & 1960 & 1965 & 1971 & 1977 & 1979 & 1983 \\
\hline \multicolumn{2}{|c|}{ I. CONSUMO HUMANO INTERNO } & 82.7 & 82.6 & 59.9 & 75.1 & 64.9 & 76.5 \\
\hline \multirow[t]{5}{*}{ I.1. } & Alimentos básicos & 68.8 & 73.3 & 51.8 & 64.2 & 56.5 & 60.4 \\
\hline & Maiz & 39.2 & 32.4 & 22.7 & 33.5 & 31.5 & 36.1 \\
\hline & Frijol & 20,4 & 33.2 & 17.1 & 21.2 & 17.4 & 17.2 \\
\hline & Trigo & 9.2 & 7.7 & 14.6 & 9.5 & 7.6 & 7.1 \\
\hline & Arroz & - & - & - & - & - & - \\
\hline \multirow[t]{9}{*}{1.2.} & $\begin{array}{l}\text { Transformación } \\
\text { Industrial }\end{array}$ & 12.3 & 6.6 & 6.3 & 8.5 & 5.9 & 13,8 \\
\hline & a) Oleaginusas & 0.3 & - & 0.9 & 2.3 & 3.5 & 1.6 \\
\hline & Ajonjolí & - & - & - & - & - & - \\
\hline & Cártamo & - & - & 0.2 & 0.1 & 0.2 & - \\
\hline & Soya & - & - & 0.7 & 2.2 & 3.3 & 1.6 \\
\hline & b) Ctros: & 12.0 & 6.6 & 5.4 & 6.2 & 2.4 & 12.2 \\
\hline & Avena grano & 11.9 & 6.6 & 5.4 & 0.1 & 2,2 & 11.8 \\
\hline & Cebada & 0.1 & - & - & 0.1 & 0.2 & 0.4 \\
\hline & Caña de azúcar & - & - & - & - & - & - \\
\hline \multirow[t]{9}{*}{1.3.} & Consumo directo & 1.6 & 2.7 & 1.8 & 2.4 & 2.5 & 2,3 \\
\hline & Cacahuate & 0.3 & 1.4 & 0.9 & 0.8 & 0.7 & 1.0 \\
\hline & Papa & 0.9 & 0.8 & 0.3 & 0.6 & 0.9 & 0.8 \\
\hline & Cebolla & - & 0.1 & 0.2 & 0.2 & 0.4 & 0.2 \\
\hline & Chícharo & $\overline{0}$ & $\overline{0}$ & - & - & $\overline{-}$ & - \\
\hline & Chile verde & 0.2 & 0.2 & 0.2 & 0.7 & 0.5 & 0.3 \\
\hline & Chile seco & 0.1 & $=$ & 0.2 & - & - & - \\
\hline & Camote & $\overline{0}$ & 0.1 & - & 0.1 & - & - \\
\hline & Sandía & 0.1 & 0.1 & - & 0.1 & - & - \\
\hline \multicolumn{2}{|c|}{ II. EXPORTACION } & 15.8 & 13.9 & 4.3 & 4.9 & 5.7 & 3.1 \\
\hline & Algodón & 15.6 & 13.7 & 4.1 & 4.8 & 5.6 & 3.1 \\
\hline & Jitomate & 0.1 & 0.1 & 0.2 & 0.1 & 0.1 & - \\
\hline & Henequén & - & - & - & - & - & - \\
\hline & Tabaco & - & $\bar{z}$ & - & - & - & - \\
\hline & $\begin{array}{l}\text { Garbanzo } \\
\text { Melón }\end{array}$ & $\overline{0.1}$ & $\overline{0.1}$ & $\overline{-}$ & $\overline{-}$ & $\bar{z}$ & - \\
\hline & Café & - & - & - & - & - & - \\
\hline \multicolumn{2}{|c|}{ III. CONSUMO ANIMAL } & 1.4 & 3.3 & 4.8 & 15.5 & 24.8 & 17.9 \\
\hline & Sorgo & 0.1 & 0.9 & 2.9 & 3.4 & 2.6 & 1.7 \\
\hline & Alfalfa & 1.3 & 2.4 & 1.9 & 3.2 & 3.8 & 3.1 \\
\hline & Avena forrajera & - & - & - & 8.5 & 17.1 & 10.4 \\
\hline & Garbanzo forrajero & - & - & - & - & 0.2 & 0.3 \\
\hline & Sorgo forrajero & - & - & - & $\overline{0}$ & 0.2 & 0.3 \\
\hline & Cebada forrajera & - & - & - & 0.3 & $\overline{0}$ & - \\
\hline & Maíz forrajero & $\overline{-}$ & $\overline{-}$ & $\overline{-}$ & 0.1 & 0.9 & 1.7 \\
\hline & $\begin{array}{l}\text { Pastos } \\
\text { Subtotal }\end{array}$ & 99.9 & 99.8 & $6 \overline{9.0}$ & $9 \overline{5.5}$ & $\begin{array}{r}0,2 \\
95.4^{\prime}\end{array}$ & $\begin{array}{r}0.7 \\
97.5\end{array}$ \\
\hline & Subtotal & & & 8.0 & 93.3 & 95.4 & 97.5 \\
\hline & TOTAL & 100.0 & 100.0 & 100.0 & 100.0 & 100.0 & 100.0 \\
\hline
\end{tabular}


CUADRO 15. Coahuila: patrón de cultivos (porcentajes).

\begin{tabular}{|c|c|c|c|c|c|c|c|}
\hline & & 1950 & 1965 & 1971 & 1977 & 1979 & 1983 \\
\hline \multicolumn{2}{|c|}{ I. CONSUMO HUMANO INTERNO } & 57.4 & 62.4 & 30.8 & 41.1 & 35.0 & 41.8 \\
\hline \multirow{5}{*}{ I.1. } & Alimentos básicos & 56.0 & 61.4 & 26.0 & 34.7 & 27.1 & 35.8 \\
\hline & Maíz & 15.6 & 18.2 & 8.2 & 18.8 & 15.6 & 20.6 \\
\hline & Frijol & 1.1 & 1.8 & 2.5 & 3.8 & 2.6 & 4.2 \\
\hline & Trigo & 39.3 & 41.4 & 5.0 & 12.1 & 8.9 & 11.0 \\
\hline & Arroz & - & - & - & - & - & - \\
\hline \multirow[t]{10}{*}{ I.2. } & Transformacion & & & & & & \\
\hline & Industrial & 1.0 & 0.7 & 4.3 & 5.9 & 7.0 & 4.4 \\
\hline & a) Oleaginosas & 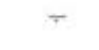 & 0.5 & 3.6 & 2.2 & 4.5 & 2.6 \\
\hline & Ajonjoli & - & - & - & - & - & - \\
\hline & Cártamo & - & - & 3.6 & 2.2 & 4.5 & 2.6 \\
\hline & Soya & - & - & - & - & - & - \\
\hline & b) Otros: & 1.0 & 0.2 & 0.7 & 3.7 & 2.5 & 1.8 \\
\hline & Avena grano & & -7 & 0.1 & 0.5 & 0.8 & 0.1 \\
\hline & Cebada & 1.0 & 0.2 & 0.6 & 3.2 & 1.7 & 1.7 \\
\hline & Caña de azúcar & - & - & - & - & -1 & - \\
\hline \multirow{9}{*}{ I.3. } & Consumo directo & 0.4 & 0.3 & 0.5 & 0.5 & 0.9 & 1.6 \\
\hline & Cacahuate & - & - & - & - & & - \\
\hline & Papa & $\overrightarrow{0}$ & - & 0.1 & - & 0.4 & 0.7 \\
\hline & Cebolla & 0.1 & - & - & 0.1 & - & 0.1 \\
\hline & Chícharo & - & - & - & - & - & - \\
\hline & Chile verde & 0.1 & - & 0.2 & 0.1 & 0.3 & 0.2 \\
\hline & Chile seco & - & - & - & - & - & - \\
\hline & Camote & - & - & - & - & $x_{0}$ & - \\
\hline & Sandía & 0.2 & 0.3 & 0.2 & 0.3 & 0.2 & 0.6 \\
\hline \multicolumn{2}{|c|}{ I.EXPORTACION } & 38.6 & 30,0 & 26.0 & 22.2 & 26.5 & 17.2 \\
\hline & Algodon & 38.1 & 29.5 & 25.8 & 21.9 & 25.9 & 16.3 \\
\hline & Jitomate & 0.4 & 0.3 & 0.1 & 0.2 & 0.2 & 0.1 \\
\hline & Henequén & - & - & - & - & - & - \\
\hline & Tabaco & - & - & - & - & - & - \\
\hline & Garbanzo & - & - & - & - & - & - \\
\hline & Melón & 0,1 & 0.2 & 0.1 & 0.1 & 0.4 & 0.8 \\
\hline & Café & - & - & - & - & - & - \\
\hline \multicolumn{2}{|c|}{ II. CONSUMO ANIMAL } & 1.6 & 3.4 & 10.4 & 20.8 & 25.1 & 26.7 \\
\hline & Sorgo & 0.6 & 1.8 & 5.9 & 6.7 & 4.8 & 5.8 \\
\hline & Alfalfa & 1.1 & 1.6 & 2.5 & 4.1 & 6.8 & 7.5 \\
\hline & Avena forrajera & & - & - & 3.6 & 5.4 & 4,9 \\
\hline & Garbanzo forrajero & - & - & - & - & - & - \\
\hline & Sorgo forrajero & - & - & 0.7 & 1.7 & 3.8 & 3.6 \\
\hline & Cebada forrajera & - & - & 1.3 & 3.4 & 0.5 & 0.5 \\
\hline & Maíz forrajero & - & - & - & 1.3 & 1.2 & 0.8 \\
\hline & Pastos & - & - & & - & 26 & 3.6 \\
\hline & Subtotal & 97.6 & 95.8 & 67.2 & 84. & 86.6 & 85.7 \\
\hline & TOTAL & 100.0 & 100,0 & 100,0 & 100.0 & 100.0 & 100.0 \\
\hline
\end{tabular}


CUADRO 16. Nuevo León: patrón de cultivos (porcentajes).

\begin{tabular}{lcccccr}
\hline & 1960 & 1965 & 1971 & 1977 & 1979 & 1983 \\
\hline 1. CONSUMO HUMANO INTERNO & 72.0 & 93.1 & 50.6 & 47.5 & 40.4 & 52.5 \\
I.1. Alimentos básicos & 69.2 & 92.1 & 48.8 & 46.5 & 38.4 & 51.6 \\
Maí & 55.8 & 74.5 & 34.4 & 37.5 & 26.3 & 30.6 \\
Frijol & 3.5 & 4.0 & 1.8 & 5.0 & 1.9 & 4.1 \\
Trigo & 9.9 & 13.6 & 12.6 & 4.0 & 10.2 & 16.9 \\
Arroz & - & - & - & - & - & -
\end{tabular}

1.2. Transformación Industrial

a) Oleaginosas

Ajonjolí

Cártamo

Soya

b) Otros:

Avena grano

Cebada

Caña de azúcar

$\begin{array}{llllll}1.4 & 0.2 & 0.6 & 02 . & 1.4 & 0.2 \\ - & - & - & - & 0.7 & - \\ - & - & - & - & - & - \\ - & - & - & - & 0.4 & - \\ - & - & - & - & 0.3 & - \\ 1.4 & 0.2 & 0.6 & 0.2 & 0.7 & 0.2 \\ 0.1 & - & - & - & - & - \\ 0.2 & 0.2 & 0.5 & 0.2 & 0.7 & 0.2 \\ 0.1 & - & 0.1 & - & - & -\end{array}$

1.3. Consumo directo

$\begin{array}{ll}1.4 & 0.8\end{array}$

1.2

0.8

0.6

0.7

Cacahuate

Papa

$\overline{1.1} \quad \overline{0.6}$

Cebolla

Chícharo

Chile verde

Chile seco

Camote

Sandra

$0.2 \quad 0.1$

- $\quad-$

$\begin{array}{ll}- & - \\ - & -\end{array}$

$\overline{0.1} \quad \overline{0.1}$

1.0

0.2

0.6

0.6

$-$

10.2

-

0.1

0.1

$-$

$-$

\section{II.EXPORTACION}

$$
\begin{aligned}
& \text { Algodón } \\
& \text { Jitomate } \\
& \text { Henequén } \\
& \text { Tabaco } \\
& \text { Garbanzo } \\
& \text { Melón } \\
& \text { Café }
\end{aligned}
$$

III. CONSUMO ANIMAL

\section{Sorgo}

Alfalfa

Avena forrajera

Garbanzo forrajero

Sorgo forrajero

Cebada forrajera

Maíz forrajero

Pastos

Subtotal

TOTAL
9.6

9.3

0.1

0.

0.2

0.2

-

0.8

0.8

$-$

$\overline{-}$

$\bar{z}$

I

- $\quad-$

$82.4 \quad 97.6$

100.0

0.2

$-$

$-$

5.8
0.7

0.5

5.8

10.7

21.7

26.0

21.0

10.3

-

19.2

1.2

19.3

2.0

15.5

- 0.2

$\overline{0.4}$

$-$

-

0.3

0.8

0.8

0.7

0.7

$-$

0.9

1.6

61.5

69.2

66.5

73.6

$100.0 \quad 100.0$

100.0

100.0 
CUADRO 17. Tamaulipas: patrón de cultivos (porcentajes).

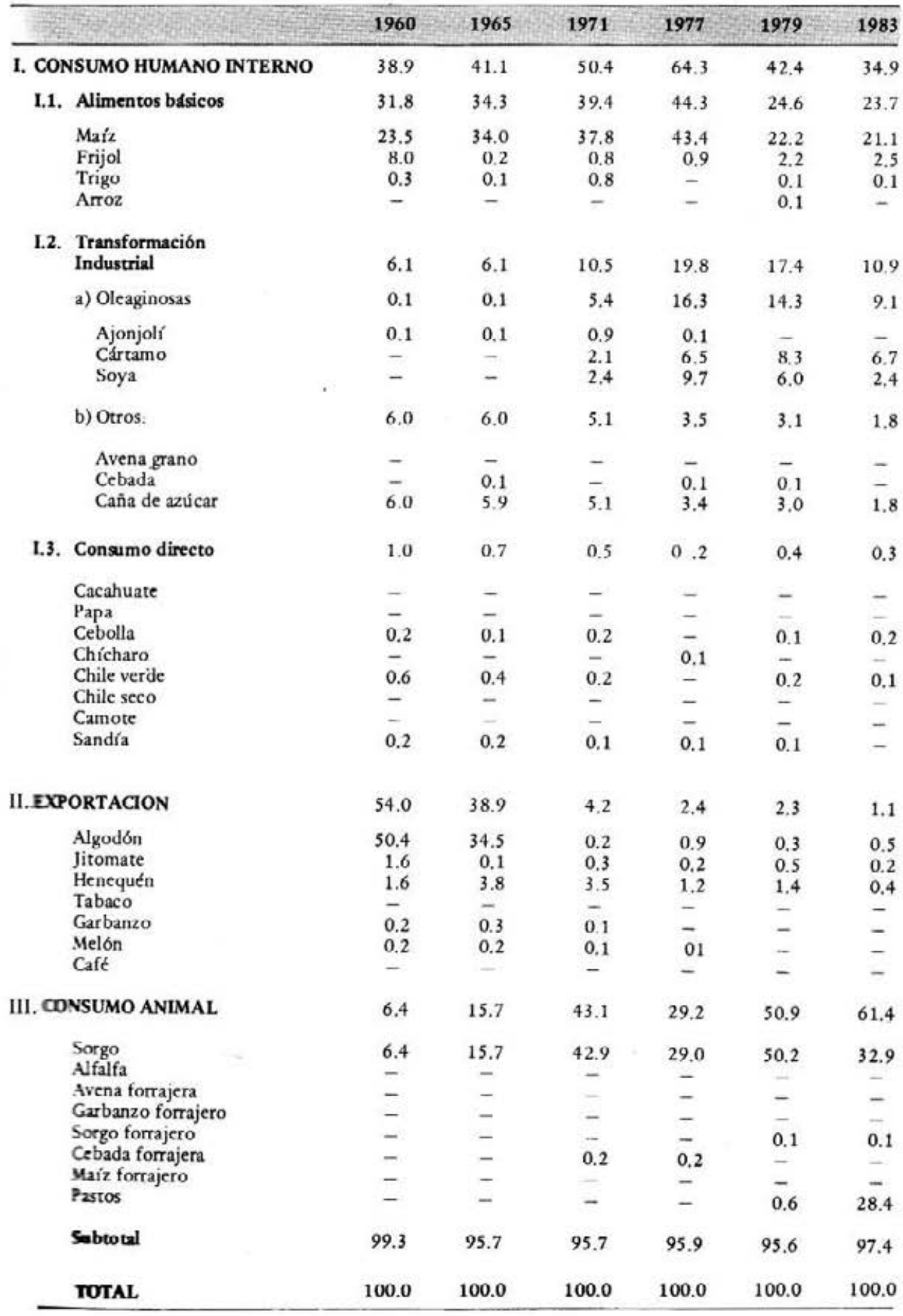


CUADRO 18. Baja California: tasas medias de crecimiento anual de la superficie cosechada.

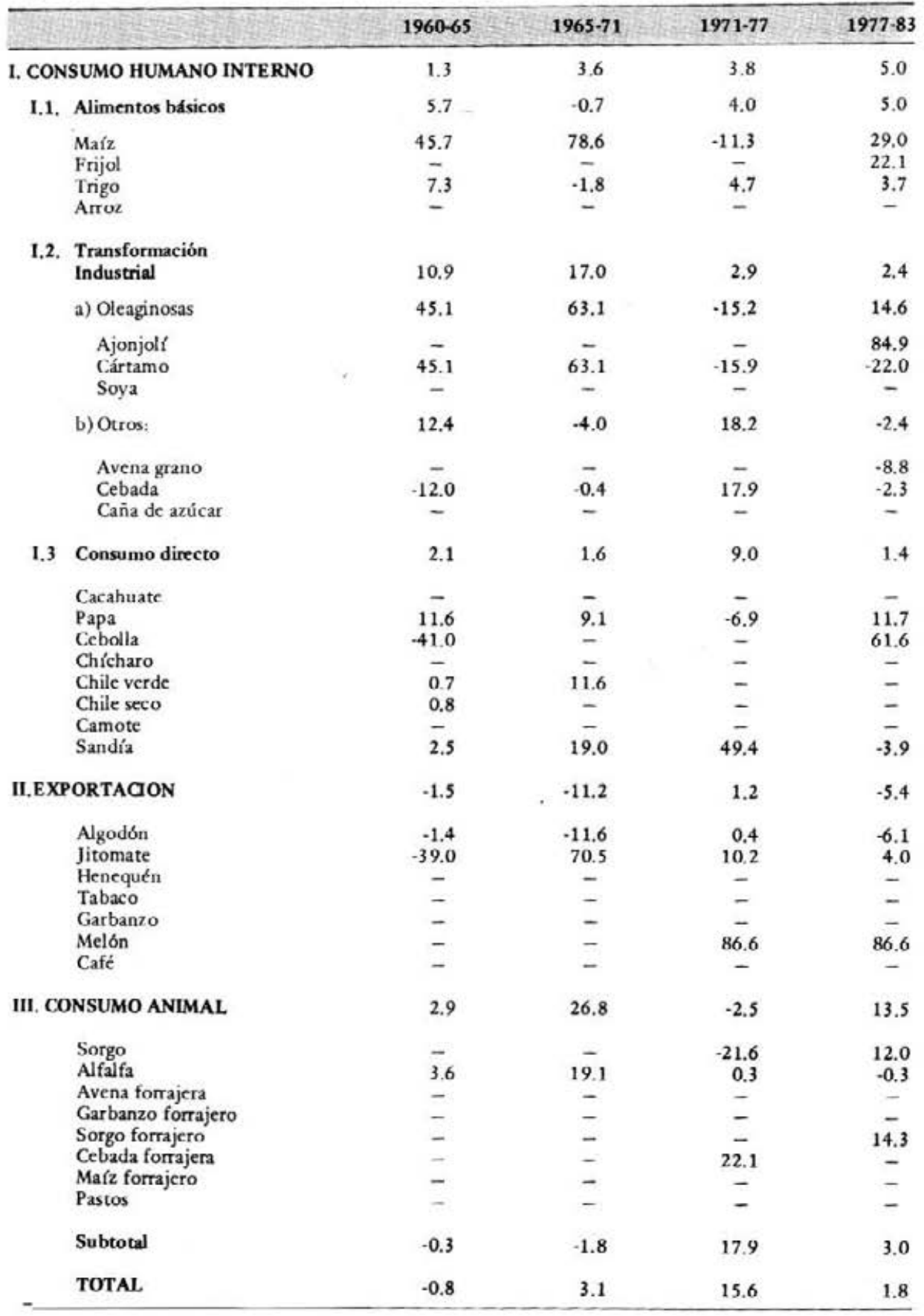


CUADRO 19. Sonora: tasas medias de crecimiento anual de la superficie cosechada.

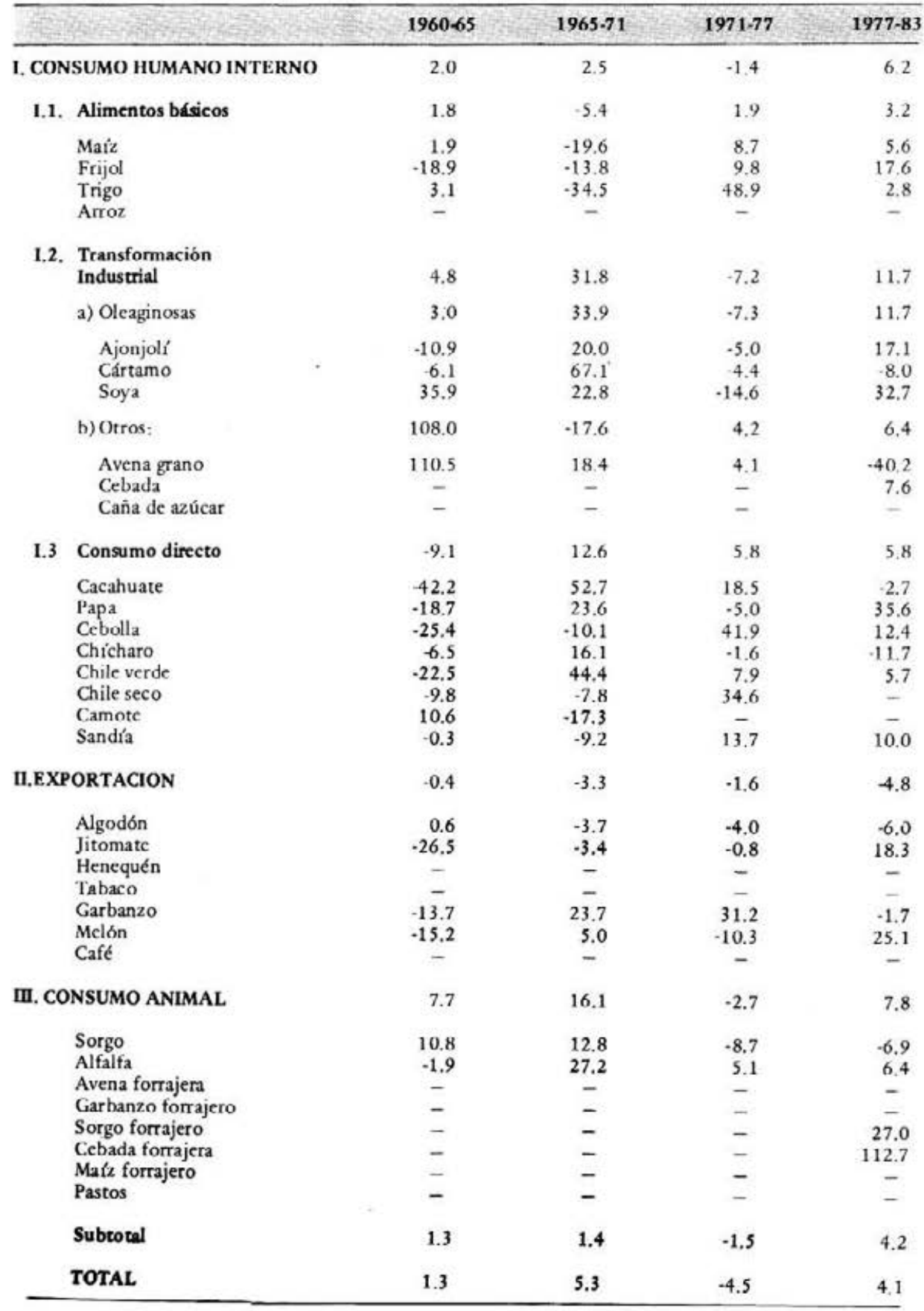


CUADRO 20. Chihuahua: tasas medias de crecimiento anual de la superficie cosechada.

\begin{tabular}{|c|c|c|c|c|c|}
\hline & & $1960-65$ & $1965-71$ & $1971-77$ & 1977.83 \\
\hline I. CON: & SUMO HUMANO INTERNO & -5.5 & 2,7 & 2.5 & 5,8 \\
\hline I.1. & Alimentos búsicos & -4.2 & -2.7 & 7.5 & 4.4 \\
\hline & $\begin{array}{l}\text { Maíz } \\
\text { Frijol }\end{array}$ & $\begin{array}{r}-9.0 \\
4.2\end{array}$ & $\begin{array}{r}2.2 \\
-3.0\end{array}$ & $\begin{array}{l}5.3 \\
2.3\end{array}$ & $\begin{array}{l}6.8 \\
1.8\end{array}$ \\
\hline & Trigo & -8.7 & 16.7 & -5.1 & 0.5 \\
\hline & Arroz & - & - & - & - \\
\hline 1.2 & $\begin{array}{l}\text { Transformacion } \\
\text { Industrial }\end{array}$ & $-16,6$ & 7.4 & 4.0 & \\
\hline & a) Oleaginosas & 26.0 & 62.9 & 16.9 & -1.5 \\
\hline & Ajonjolí & -13.7 & 3.8 & - & - \\
\hline & Cártamo & -26.3 & 23.9 & -5.0 & - \\
\hline & Soya & - & - & 20.0 & 0.7 \\
\hline & b) Otros: & -16.4 & 5.0 & 0.9 & 18.3 \\
\hline & Avena grano & -16.3 & 5.0 & 0.6 & 18.0 \\
\hline & Cebada & $\overline{-}$ & - & - & 29.6 \\
\hline & Cana de azucar & -13.2 & - & - & - \\
\hline 1.3 & Consumo directo & 4.2 & 1.5 & 3.1 & 5.2 \\
\hline & Cacahuate & 25.5 & 0.8 & -2.8 & 8.4 \\
\hline & Papa & $\begin{array}{r}-7.4 \\
140\end{array}$ & $\begin{array}{r}-5.6 \\
17.5\end{array}$ & 7.2 & 11.7 \\
\hline & $\begin{array}{l}\text { Cebolla } \\
\text { Chícharo }\end{array}$ & $\begin{array}{r}14.0 \\
.13 .2\end{array}$ & $\begin{array}{r}17.5 \\
2.0\end{array}$ & 4.6 & 6.6 \\
\hline & Chile verde & 3.5 & $\begin{array}{l}2.0 \\
4.7\end{array}$ & $2 \overline{3.6}$ & -7.8 \\
\hline & Chile seco & -36.9 & 60.9 & - & - \\
\hline & Camote & 14.8 & .6 .9 & 0.5 & - \\
\hline & Sandía & -3.3 & -8.7 & 3,1 & -35.3 \\
\hline II. EXPC & ORTACION & -7.8 & $\cdot 11.0$ & 48.5 & -2.6 \\
\hline & Algodón & -7.9 & -11.5 & 1.3 & -2.1 \\
\hline & Jitomate & -5.9 & 18.8 & -1.7 & -26.7 \\
\hline & Henequén & - & $\overline{-}$ & - & - \\
\hline & Tabaco & $\overline{2}$ & $\overline{0.8}$ & - & $\overline{-}$ \\
\hline & $\begin{array}{l}\text { Garbanzo } \\
\text { Melón }\end{array}$ & $\begin{array}{l}-3.1 \\
-2.8\end{array}$ & $\begin{array}{r}0.8 \\
-9.1\end{array}$ & 12.2 & $-\overline{14.3}$ \\
\hline & Café & $-2,8$ & - & - & - \\
\hline III. COI & NSUMO ANIMAL & 12.4 & 15.7 & 19.6 & 8.0 \\
\hline & Sorgo & 45.7 & 32.7 & 0,6 & -5.2 \\
\hline & Alfalfa & 7.3 & 4.4 & 7.2 & 4.7 \\
\hline & Avena forrajera & - & - & - & 9.1 \\
\hline & Garbanzo forrajero & $\bar{z}$ & - & - & - \\
\hline & Sorgo forrajero & $\overline{-}$ & $\bar{z}$ & - & 4.9 \\
\hline & Cebada forrajera & $\bar{z}$ & z & - & -33.7 \\
\hline & $\begin{array}{l}\text { Maíz forrajero } \\
\text { Pastos }\end{array}$ & $\bar{z}$ & - & $\bar{z}$ & 62.5 \\
\hline & & & & & - \\
\hline & Subtotal & -5.5 & -30.6 & 4.2 & 5.8 \\
\hline & TOTAL & -5.5 & 8.3 & -1.3 & 5.5 \\
\hline
\end{tabular}


CUADRO 21. Coahuila: tasas medias de crecimiento anual de la superficie cosechada.

\begin{tabular}{|c|c|c|c|c|c|c|}
\hline & & & $1960-65$ & $1965-71$ & 1971-77 & 1977.83 \\
\hline I. $\mathrm{CON}$ & USUMO HUMANO IN & RNO & 3.2 & -11.5 & 6.9 & -2.8 \\
\hline I.1. & Alimentos bisicos & & 3.4 & -13.7 & 6.8 & -2.6 \\
\hline & Maiz & & 4.6 & -12.7 & 16.8 & $-1,5$ \\
\hline & Frijol & & 10.9 & 5.6 & 9.3 & -1.5 \\
\hline & Trigo & & 2.6 & -15.6 & -2.0 & -4.6 \\
\hline & Arroz & & - & - & - & - \\
\hline 1.2. & $\begin{array}{l}\text { Transformación } \\
\text { Industrial }\end{array}$ & & & & & \\
\hline & Industrial & & -6.0 & 35.3 & 7.2 & -7.4 \\
\hline & a) Oleaginosas & & 78.2 & 38.7 & -6.0 & -0.2 \\
\hline & Ajonjolí & & -2.6 & $4 \overline{0.1}$ & $\overrightarrow{6.0}$ & $\overline{-0.2}$ \\
\hline & $\begin{array}{l}\text { Cártamo } \\
\text { Soya }\end{array}$ & & - & $\begin{array}{c}40.1 \\
-\end{array}$ & - & -0.2 \\
\hline & b) Otros: & & 26.6 & 23.1 & 34.4 & -13.8 \\
\hline & Avena grano & & - & - & 33.9 & -25.9 \\
\hline & Cebada & & -25.8 & 19.9 & 34.5 & -12.5 \\
\hline & Caña de azúcar & & - & - & - & - \\
\hline 1.3 & Consumo directo & & -0.6 & 4.7 & 3.5 & 17.0 \\
\hline & Cacahuate & & - & - & - & - \\
\hline & Papa & & -2.5 & 23.9 & -13.1 & 63.5 \\
\hline & Ccbolla & & -15.8 & -0.3 & 10.5 & $-12,0$ \\
\hline & Chicharo & & - & - & - & - \\
\hline & Chile verde & & - & - & -5.3 & -2.8 \\
\hline & Chile seco & $>$ & $-1 \overline{6} 5$ & $\overline{-}$ & $\overline{-}$ & $\overline{-}$ \\
\hline & $\begin{array}{l}\text { Camote } \\
\text { Sandŕa }\end{array}$ & & $\begin{array}{r}-16.5 \\
12.1\end{array}$ & $-1 \overline{2.3}$ & 16.2 & $1 \overline{1.2}$ \\
\hline П.EXP & ORTACION & & -3.5 & -2.8 & -0.8 & -7.1 \\
\hline & Algodón & & -3.5 & -2.6 & .0 .9 & .7 .6 \\
\hline & Jitomate & & -2.7 & $-12,4$ & 9.4 & 21.5 \\
\hline & Henequén & & - & - & - & - \\
\hline & Tabaco & & -. & - & $\overline{-}$ & - \\
\hline & Garbanzo & & $\overline{5}$ & 0.6 & $\overline{0.7}$ & - \\
\hline & Melón & & 15.2 & - & 0.7 & 33.6 \\
\hline & & & & & & \\
\hline III. CO! & NSUMO ANIMAL & & 17.4 & 76.1 & 14.3 & 1.1 \\
\hline & Sorgo & & 31.0 & 20.6 & 4.0 & -5.2 \\
\hline & Alfalfa & & 7.9 & 8.1 & 10.7 & 7.3 \\
\hline & Avena forrajera & & - & - & - & 2.4 \\
\hline & Garbanzo forrajero & & - & - & - & - \\
\hline & Sorgo forrajero & & - & - & 19.5 & -2.0 \\
\hline & Cebada forrajera & & - & - & 18.6 & -21.4 \\
\hline & Maíz forrajero & & - & - & - & -10.8 \\
\hline & Pastos & & - & - & $\rightarrow$ & - \\
\hline & Subtotal & & 1.1 & -6.8 & 6.5 & -2.7 \\
\hline & TOTAL & & 1.5 & -0.3 & 1.8 & -3.0 \\
\hline
\end{tabular}


CUADRO 22. Nuevo León: tasas medias de crecimiento anual de la superficie cosechada.

\begin{tabular}{|c|c|c|c|c|c|}
\hline \multirow{2}{*}{\multicolumn{2}{|c|}{ 1. CONSUMO HUMANO INTERNO }} & \multirow{2}{*}{$\frac{1960-65}{90}$} & \multirow{2}{*}{$\begin{array}{l}1965 \cdot 71 \\
-15.6\end{array}$} & \multirow{2}{*}{$\frac{1971-77}{6.8}$} & \multirow{2}{*}{$\frac{1977 \cdot 83}{1.0}$} \\
\hline & & & & & \\
\hline I.1. & Alimentos básicos & 9.7 & -15.9 & 7.1 & 1.1 \\
\hline & Maiz & 9.7 & -17.8 & 9.5 & $-4,0$ \\
\hline & Frijol & 7.0 & -18.5 & 28.0 & -3.7 \\
\hline & Trigo & 10.3 & -7.7 & -109 & 26.5 \\
\hline & Arrol & - & - & - & - \\
\hline \multirow{10}{*}{1.2} & Transformación & & & & \\
\hline & Industrial & -30.8 & 15.4 & $-9,1$ & -1.9 \\
\hline & a) Oleaginosas & - & - & 51.3 & -3.0 \\
\hline & Ajonjolí & - & - & - & - \\
\hline & Cártamo & - & - & - & - \\
\hline & Soya & - & - & - & - \\
\hline & b) Otros: & -30.8 & 15.3 & -10.8 & -1.8 \\
\hline & Avena grano & - & - & - & - \\
\hline & Cebada & -26.3 & 13.2 & - $\quad-9.2$ & -3.3 \\
\hline & Caña de azúcar & - & - & - & - \\
\hline \multirow[t]{9}{*}{ I. 3} & Consumo directo & 8.1 & - & 1.3 & -3.5 \\
\hline & Cacahuate & - & - & - & - \\
\hline & Papa & -9.1 & 2.9 & -0.5 & -2.2 \\
\hline & Cebolla & -19.9 & 7.3 & -12.3 & -44.3 \\
\hline & Chícharo & -12.9 & - & - & - \\
\hline & Chile verde & - & - & - & 41.1 \\
\hline & Chile seco & - & - & - & - \\
\hline & Camote & -4.4 & - & - & - \\
\hline & Sandía & 25.9 & -37.8 & 30.8 & 19.8 \\
\hline \multicolumn{2}{|c|}{ II.EXPORTACION } & -39.2 & . $\quad-26.4$ & -15.3 & 18.3 \\
\hline & Algodón & -42.2 & -49.5 & - & - \\
\hline & Jitomate & 8.9 & -9.3 & -18.2 & -6.5 \\
\hline & Henequén & - & - & - & - \\
\hline & Tabaco & - & - & - & - \\
\hline & Garbanzo & 11.8 & - & - & - \\
\hline & Melón & 6.8 & -21.2 & 30.8 & 38.0 \\
\hline & Cafć & - & - & - & - \\
\hline \multicolumn{2}{|c|}{ III. CONSUMO ANIMAL. } & 55.5 & 3.6 & 21.3 & -1.2 \\
\hline & Sorgo & 55.5 & 3.0 & -19.7 & -4.2 \\
\hline & Alfalfa & - & - & - & -2.7 \\
\hline & Avena forrajera & - & - & - & 32.7 \\
\hline & Garbaniso forrajero & - & - & - & - \\
\hline & Sorgo forrajero & - & - & 1.0 & 12.4 \\
\hline & Cebada forrajera & - & - & - & 5.1 \\
\hline & Maíz. forrajero & - & - & - & - \\
\hline & Pastes & - & - & - & - \\
\hline & Subtotal & 7.6 & -13.7 & 10.1 & 0.4 \\
\hline & TOTAL & 3.6 & -6.5 & 7.9 & -0.6 \\
\hline
\end{tabular}


CUADRO 23. Tamaulipas: tasas medias de crecimiento anual de la superficie cosechada.

\begin{tabular}{|c|c|c|c|c|c|}
\hline & & $1960-65$ & $1965-71$ & 1971.77 & $1977-83$ \\
\hline \multicolumn{2}{|c|}{ I. CONSUMO HUMANO INTERNO } & 2.7 & 6.4 & 11.6 & $-3,9$ \\
\hline \multirow[t]{5}{*}{ I.1. } & Alimentos básicos & 3.2 & 6.4 & 9.3 & -4.1 \\
\hline & Maíz & 9.4 & 4.6 & 9.7 & -5.6 \\
\hline & Frijol & -49.7 & 27.0 & 9.1 & 25.6 \\
\hline & Trigo & 19.4 & 43.2 & -43.0 & 32.5 \\
\hline & Arroz & - & - & - & - \\
\hline \multirow[t]{9}{*}{ I.2. } & $\begin{array}{l}\text { Transformación } \\
\text { Industrial }\end{array}$ & 1.3 & 12.6 & 19.1 & -3.6 \\
\hline & a) Oleaginosas & 5.4 & 90.3 & 29.0 & -3.5 \\
\hline & Ajonjolí & 5.4 & 42.7 & -27.3 & -16.7 \\
\hline & Cártamo & - & - & 30.0 & 7.0 \\
\hline & Soya & - & - & 35.6 & -15.9 \\
\hline & b) Otros: & 1.2 & 0,3 & 0.4 & -4.5 \\
\hline & Avena grano & - & - & - & - \\
\hline & Ccbada & 34.2 & - & - & -8.6 \\
\hline & Cańa de azúcar & 1.1 & 0.5 & - & -4.4 \\
\hline \multirow[t]{9}{*}{1.3} & Consumo directo & -4.6 & -2.3 & -6.2 & 12.5 \\
\hline & Cacahuate & - & - & - & - \\
\hline & Papa & -19.7 & - & - & - \\
\hline & Celoulla & $\cdot 8.9$ & 18.0 & -15.0 & 22.1 \\
\hline & Chicharo & - & - & 47.1 & -11.3 \\
\hline & Chjle verde & -3.5 & -10.0 & -11.7 & 9.5 \\
\hline & Chile seco & - & - & - & - \\
\hline & Camote & - & - & - & $\overline{-}$ \\
\hline & Sandía & 4.0 & -12.9 & 10.2 & -7.4 \\
\hline \multicolumn{2}{|c|}{ II. EXPORTACION } & -4.8 & -29.0 & -2.7 & -6.7 \\
\hline & Algodón & -5.8 & -57.4 & 41.6 & -4.3 \\
\hline & Jitomate & $-36: 9$ & 15.1 & -2.8 & 7.0 \\
\hline & Henequén & 21.0 & 1.7 & -10.4 & -12.9 \\
\hline & Tahaco & - & - & - & - \\
\hline & Garbanzo & 6.1 & -12.4 & -67.2 & - \\
\hline & Melón & 8.7 & -8.1 & -2.4 & 4.3 \\
\hline & Café & - & - & - & - \\
\hline \multicolumn{6}{|c|}{ III, CONSUMO ANIMAL } \\
\hline \multicolumn{2}{|c|}{ III. CONSUMO ANIMAL } & 21.7 & 21.6 & 0.5 & 20.4 \\
\hline & Sorgo & 21.7 & 21,5 & 0.5 & 8.6 \\
\hline & Alfalfa & - & - & - & - \\
\hline & Avena forrajera & - & - & - & 25.6 \\
\hline & Garbanzo forrajero & - & - & - & - \\
\hline & Sorgo forrajero & - & - & - & 0.3 \\
\hline & Cebada forrajera & - & - & -1.8 & - \\
\hline & Maíz forrajero & - & - & - & $\overline{-}$ \\
\hline & Pastos & - & - & - & - \\
\hline & Subtotal & 0.9 & 3.2 & 6.8 & 6.7 \\
\hline & TOTAL. & 1.6 & 2.8 & 7.6 & 6.4 \\
\hline
\end{tabular}

